UDC 546:548.736.4

\author{
N. Klymentiy ${ }^{a}$, S. Pukas ${ }^{a}$, L. Orovčík ${ }^{b}$, R. Gladyshevskii ${ }^{a}$
}

\title{
REINVESTIGATION OF THE SYSTEM Ti-Cu-Al AT 800
}

\author{
a Ivan Franko National University of Lviv, Lviv, Ukraine \\ ${ }^{b}$ Institute of Materials and Machine Mechanics, Bratislava, Slovakia
}

\begin{abstract}
The isothermal section of the phase diagram of the ternary system $\mathrm{Ti}-\mathrm{Cu}-\mathrm{Al}$ at the temperature of $800^{\circ} \mathrm{C}$ was investigated using X-ray powder diffraction, microstructural analysis and energy-dispersive $\mathrm{X}$-ray spectroscopy. The homogeneity ranges at $800^{\circ} \mathrm{C}$ of three purely ternary compounds were determined: $\mathrm{TiCu}_{\mathrm{x}} \mathrm{Al}_{3-\mathrm{x}}, \mathrm{x}=0.30(3)-0.60(3)$ (structure type $\mathrm{Cu}_{3} \mathrm{Au}$ ), $\mathrm{TiCu}_{2+\mathrm{x}} \mathrm{Al}_{1-\mathrm{x}}, \mathrm{x}=0-0.52(2)$ (structure type $\mathrm{MnCu}_{2} \mathrm{Al}$ ) and $\mathrm{TiCu}_{\mathrm{x}} \mathrm{Al}_{2-\mathrm{x}}$, $\mathrm{x}=0.54(3)-1.14(3)$ (structure type $\mathrm{MgZn}_{2}$ ). The crystal structure of an additional compound near the composition $\mathrm{TiAl}_{3}$ was refined on $\mathrm{X}$-ray powder diffraction data $\left(\mathrm{Ti}_{1.15(2)} \mathrm{Cu}_{0.09(1)} \mathrm{Al}_{2.76(3)}\right.$, structure type $\mathrm{ZrAl}_{3}$; Pearson symbol $t I 16$, space group $14 / \mathrm{mmm}$, $\mathrm{a}=3.9159(3), \mathrm{c}=16.556(2) \AA)$. This ternary compound, observed at $800^{\circ} \mathrm{C}$, is probably an extension of the solid solution of the binary high-temperature phase reported as $\mathrm{Ti}_{5} \mathrm{Al}_{11}$ with the same structure type. The microhardness of the ternary compounds was measured.
\end{abstract}

Keywords: aluminum, copper, titanium, X-ray powder diffraction, phase diagram, ternary compound, microstructure, hardness.

DOI: $10.32434 / 0321-4095-2019-125-4-62-72$

\section{Introduction}

Literature data on the $\mathrm{Ti}-\mathrm{Cu}-\mathrm{Al}$ system has been summarized by Ran and Stadelmaier [1]. The existence of a ternary aluminide with equiatomic composition in the system $\mathrm{Ti}-\mathrm{Cu}-\mathrm{Al}$ was established already in 1935 and a cell corresponding to the hexagonal Laves type $\mathrm{MgZn}_{2}$ was reported; thirty years later $\mathrm{TiCu}_{2} \mathrm{Al}$ with $\mathrm{CsCl}$-type structure was reported, and cell parameters for the same phase were published [2,3]. Three compounds, $\mathrm{TiCu}_{0.08} \mathrm{Al}_{2.92}, \mathrm{TiCu}_{0.12} \mathrm{Al}_{2.88}$ and $\mathrm{TiCu}_{0.16} \mathrm{Al}_{2.84}$, with different variants of cubic close-packing (structure types $\mathrm{TiAl}_{3}, \mathrm{Cu}_{3} \mathrm{Au}$ and $\mathrm{ZrAl}_{3}$, respectively), were reported close to the boundary system $\mathrm{Ti}-\mathrm{Al}$, and the structure of the compound $\mathrm{TiCu}_{2.25} \mathrm{Al}_{0.75}$ was assigned the type $\mathrm{MnCu}_{2} \mathrm{Al}$, commonly referred to as Heusler phase [4]. The latter appears to be the same as the compound earlier reported as $\mathrm{TiCu}_{2} \mathrm{Al}$, the $\mathrm{MnCu}_{2} \mathrm{Al}$ type being an ordered derivative of the $\mathrm{CsCl}$ type.

The interaction of the components in the system $\mathrm{Ti}-\mathrm{Cu}-\mathrm{Al}$ at $500^{\circ} \mathrm{C}$ [5], $540^{\circ} \mathrm{C} \mathrm{[6]} \mathrm{and} 800^{\circ} \mathrm{C}$ [5] has been investigated in almost the whole concentration range (Figs. 1-3). The authors confirmed the existence of three ternary compounds with idealized compositions $\mathrm{Ti}_{2} \mathrm{CuAl}_{5}\left(\mathrm{TiCu}_{0.5} \mathrm{Al}_{2.5}\right.$, structure type $\left.\mathrm{Cu}_{3} \mathrm{Au}\right), \mathrm{TiCu}_{2} \mathrm{Al}\left(\mathrm{MnCu}_{2} \mathrm{Al}\right)$, and $\mathrm{TiCuAl}\left(\mathrm{MgZn}_{2}\right)$ and determined their homogeneity ranges. According to Virdis and Zwicker [6], at $540^{\circ} \mathrm{C}$ all three compounds can have variable Ti content, from 5 at.\% $\left(\mathrm{Ti}_{2} \mathrm{CuAl}_{5}\right.$ and $\left.\mathrm{TiCuAl}\right)$ to 9 at.\% $\left(\mathrm{TiCu}_{2} \mathrm{Al}\right)$, whereas according to Markiv et al. [5] only one of the compounds, $\mathrm{TiCu}_{2} \mathrm{Al}$, can contain more or less $\mathrm{Ti}$ (4 at.\%). The compound $\mathrm{TiCuAl}$ was reported to have a homogeneity range along the isoconcentrate 40 at.\% $\mathrm{Ti}$ from 30 to 40 at.\% $\mathrm{Al}$ at $540^{\circ} \mathrm{C}$ [6], but according to Markiv et al. [5], at $500^{\circ} \mathrm{C}$ this compound is located along the isoconcentrate 33.3 at.\% $\mathrm{Ti}$ and extends from 35 to 44 at.\% Al. In both works, the cubic close-packed phase $\mathrm{Ti}_{2} \mathrm{CuAl}_{5}$ and the Heusler phase $\mathrm{TiCu}_{2} \mathrm{Al}$ are located along the isoconcentrate 25 at.\% Ti, but slightly different homogeneity ranges were found: $57-65$ at. $\% \mathrm{Al}\left(540^{\circ} \mathrm{C}\right)$ and $55-63$ at. $\% \mathrm{Al}\left(500^{\circ} \mathrm{C}\right)$ for the former, and $5-25$ at. $\% \mathrm{Al}\left(540^{\circ} \mathrm{C}\right)$ and $13-$ 25 at. $\% \mathrm{Al}\left(500^{\circ} \mathrm{C}\right)$ for the latter. For these three compounds the homogeneity ranges at $800^{\circ} \mathrm{C}$ are the same as at $500^{\circ} \mathrm{C}$, while the phase equilibria differ slightly.

Information about hardness measurements is available for some compounds in the system $\mathrm{Ti}-\mathrm{Cu}$ [7]. The hardness of the pure metals is as follows:

(C) N. Klymentiy, S. Pukas, L. Orovčík, R. Gladyshevskii, 2019 


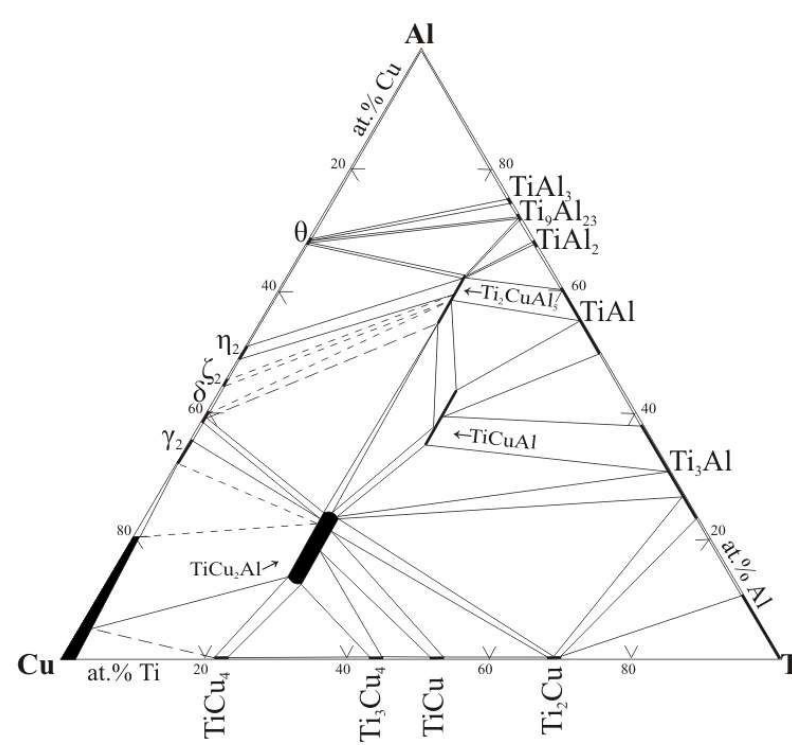

Fig. 1. Isothermal section of the phase diagram of the system $\mathrm{Ti}-\mathrm{Cu}-\mathrm{Al}$ at $500^{\circ} \mathrm{C}$ according to Markiv et al. [5]

$0.970 \mathrm{GPa}, 0.369 \mathrm{GPa}$ and $0.167 \mathrm{GPa}$ for titanium, copper and aluminum, respectively. According to ref. [7], the hardness of the compound $\mathrm{TiCu}$ is $0.370 \mathrm{GPa}$, i.e. lower than for titanium, but similar to the value for copper.

\section{Experimental}

Alloys of the system $\mathrm{Ti}-\mathrm{Cu}-\mathrm{Al}$ were synthesized from the metals (purity for $\mathrm{Ti} \geq 99.99 \mathrm{wt} . \%$, $\mathrm{Cu} \geq 99.99$ wt.\%, $\mathrm{Al} \geq 99.998$ wt.\%) by arc melting under a purified argon atmosphere. The ingots were annealed at $800^{\circ} \mathrm{C}$ under vacuum in quartz ampoules for 1 week and subsequently quenched in cold water. Phase and structural analyses were performed on X-ray powder diffraction data (diffractometers DRON 2.0M, FeK ${ }_{\alpha}$ radiation, and STOE Stadi P, $\mathrm{CuK}_{\alpha 1}$ radiation, angular range $6^{0} \leq 2 \theta \leq 108^{\circ}$, step $0.015^{\circ}$, scan time $230 \mathrm{~s}$ per step). The profile and structural parameters were refined by the Rietveld method, using the program DBWS [8].

The microstructure of the samples was studied by scanning electron microscopy (SEM, JEOL JSM-7600F equipped with an energy dispersive $\mathrm{X}$-ray (EDX) analyzer Oxford Instruments, X-max $50 \mathrm{~mm}^{2}$ ). The parameters of the microscope for sample observation in COMPO mode and EDX chemical analysis were set at $15 \mathrm{kV}$.

The hardness was measured with a Microhardness Tester (FM-100, Future-Tech Corp.). A square-based pyramidal diamond was pressed using a force of $5 \mathrm{~N}$ for a loading time of $10 \mathrm{~s}$; at least eight areas across each joint were analyzed to obtain an average value.

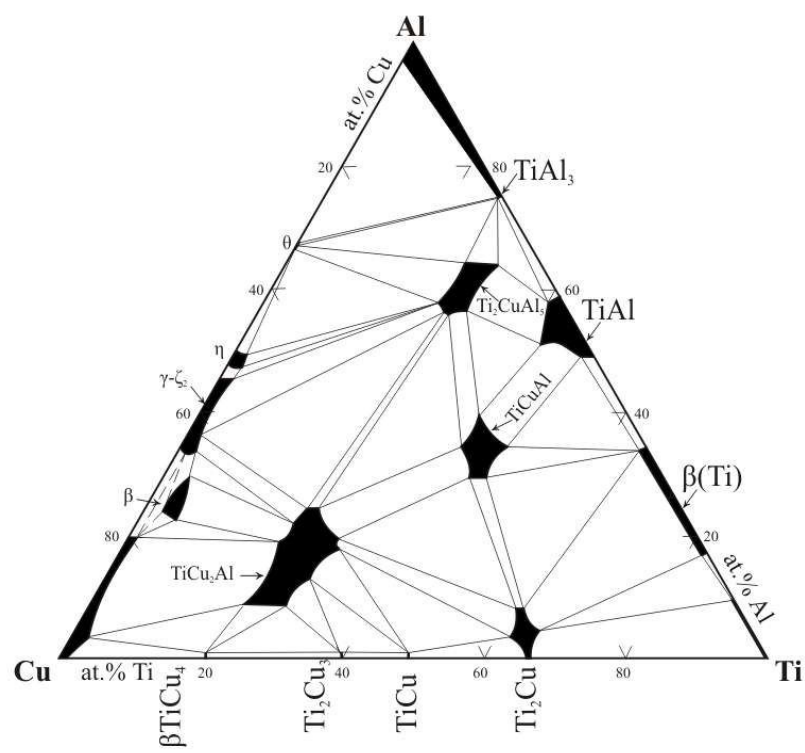

Fig. 2. Isothermal section of the phase diagram of the system $\mathrm{Ti}-\mathrm{Cu}-\mathrm{Al}$ at $540^{\circ} \mathrm{C}$ according to Virdis and Zwicker [6]

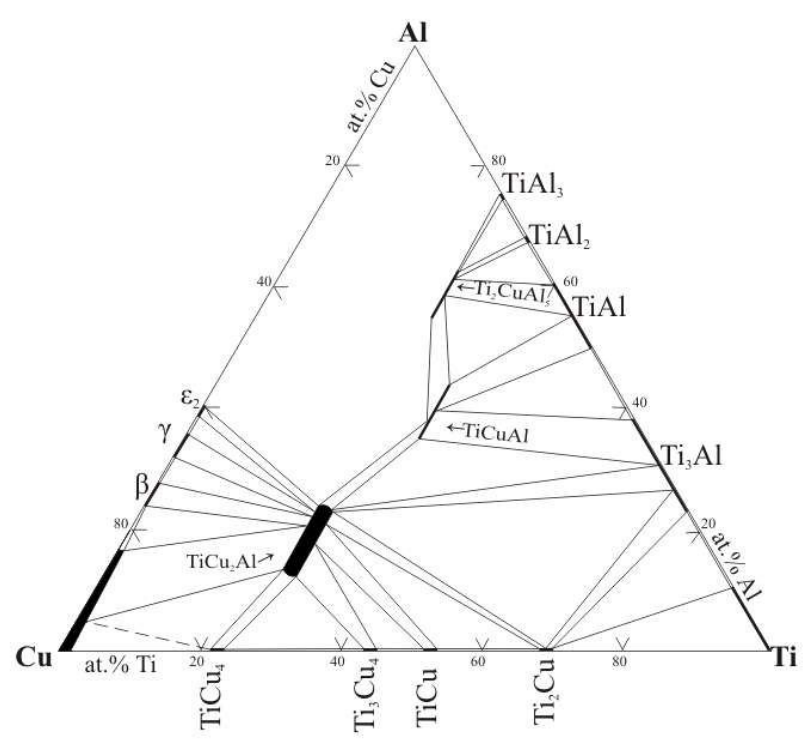

Fig. 3. Isothermal section of the phase diagram of the system $\mathrm{Ti}-\mathrm{Cu}-\mathrm{Al}$ at $800^{\circ} \mathrm{C}$ according to Markiv et al. [5]

\section{Results and discussion}

$X$-ray phase analysis

Table 1 presents the results of the phase analysis by X-ray diffraction of 2 binary and 33 ternary samples annealed at $800^{\circ} \mathrm{C}$. The unit-cell parameters of some of the binary compounds in the systems $\mathrm{Ti}-\mathrm{Cu}, \mathrm{Ti}-\mathrm{Al}$, and $\mathrm{Cu}-\mathrm{Al}\left(\mathrm{TiCu}, \mathrm{Ti}_{2} \mathrm{Cu}, \mathrm{TiAl}, \mathrm{Ti}_{3} \mathrm{Al}\right.$, $\mathrm{Cu}_{1.7} \mathrm{Al}$, and $\mathrm{Cu}_{9} \mathrm{Al}_{4}$ ) and the compositions and unitcell parameters of the four ternary compounds in the system $\mathrm{Ti}-\mathrm{Cu}-\mathrm{Al}\left(\mathrm{Ti}_{1.15(2)} \mathrm{Cu}_{0.09(1)} \mathrm{Al}_{2.76(3)}\right.$, 
Table 1

Phase composition of the samples investigated in the ternary system $\mathrm{Ti}-\mathrm{Cu}-\mathrm{Al}$

\begin{tabular}{|c|c|c|}
\hline Nominal composition of the sample & Phases $^{\mathrm{a}}$ & Structure type, space group, unit-cell parameters \\
\hline 1 & 2 & 3 \\
\hline $\mathrm{Cu}_{62.96} \mathrm{Al}_{37.04}$ & $\mathrm{Cu}_{1.7} \mathrm{Al}$ & $\mathrm{Co}_{1.75} \mathrm{Ge}, P 6_{3} / m m c, \mathrm{a}=4.108(1), \mathrm{c}=5.014(1) \AA$ \\
\hline $\mathrm{Cu}_{69.23} \mathrm{Al}_{30.77}$ & $\mathrm{Cu}_{9} \mathrm{Al}_{4}$ & $\mathrm{Cu}_{9} \mathrm{Al}_{4}, P-43 m, \mathrm{a}=8.694(1) \AA$ \\
\hline $\mathrm{Ti}_{10} \mathrm{Cu}_{50} \mathrm{Al}_{40}$ & $\begin{array}{l}\mathrm{Cu}_{9} \mathrm{Al}_{4} \\
\mathrm{TiCu}_{0.5} \mathrm{Al}_{2.5} \\
\end{array}$ & $\begin{array}{l}\mathrm{Cu}_{9} \mathrm{Al}_{4}, P-43 m, \mathrm{a}=8.7056(9) \AA \\
\mathrm{Cu}_{3} \mathrm{Au}, P m-3 m, \mathrm{a}=3.9075(7) \AA \\
\end{array}$ \\
\hline $\mathrm{Ti}_{10} \mathrm{Cu}_{60} \mathrm{Al}_{30}$ & $\begin{array}{l}\mathrm{Cu}_{9} \mathrm{Al}_{4} \\
\mathrm{TiCu}_{2} \mathrm{Al} \\
\end{array}$ & $\begin{array}{l}\mathrm{Cu}_{9} \mathrm{Al}_{4}, P-43 m, \mathrm{a}=8.704(1) \AA \\
\mathrm{MnCu}_{2} \mathrm{Al}, F m-3 m, \mathrm{a}=6.0163(8) \AA\end{array}$ \\
\hline $\mathrm{Ti}_{17} \mathrm{Cu}_{33} \mathrm{Al}_{50}$ & $\begin{array}{l}\mathrm{TiCu}_{0.5} \mathrm{Al}_{2.5} \\
\mathrm{Cu}_{9} \mathrm{Al}_{4}\end{array}$ & $\begin{array}{l}\mathrm{Cu}_{3} \mathrm{Au}, P m-3 m, \mathrm{a}=3.9138(5) \AA \\
\mathrm{Cu}_{9} \mathrm{Al}_{4}, P-43 m, \mathrm{a}=8.720(1) \AA\end{array}$ \\
\hline $\mathrm{Ti}_{20} \mathrm{Cu}_{20} \mathrm{Al}_{60}$ & $\begin{array}{l}\mathrm{TiCu}_{0.5} \mathrm{Al}_{2.5} \\
\mathrm{Cu}_{1.7} \mathrm{Al} \\
\end{array}$ & $\begin{array}{l}\mathrm{Cu}_{3} \mathrm{Au}, P m-3 m, \mathrm{a}=3.9138(5) \AA \\
\mathrm{Co}_{1.75} \mathrm{Ge}, P 6_{3} / m m c, \mathrm{a}=4.1022(1), \mathrm{c}=5.008(2) \AA\end{array}$ \\
\hline $\mathrm{Ti}_{20} \mathrm{Cu}_{60} \mathrm{Al}_{20}$ & $\begin{array}{l}\mathrm{TiCuAl} \\
\mathrm{TiCu}_{2} \mathrm{Al} \\
\mathrm{TiCu}_{0.5} \mathrm{Al}_{2.5} \\
\end{array}$ & $\begin{array}{l}\mathrm{MgZn}_{2}, P 6_{3} / m m c, \mathrm{a}=5.0402(8), \mathrm{c}=8.1073(1) \AA \\
\mathrm{MnCu}_{2} \mathrm{Al}, F m-3 m, \mathrm{a}=6.0212(1) \AA \\
\mathrm{Cu}_{3} \mathrm{Au}, P m-3 m, \mathrm{a}=3.9077(7) \AA\end{array}$ \\
\hline $\mathrm{Ti}_{25} \mathrm{Cu}_{6.25} \mathrm{Al}_{68.75}$ & \begin{tabular}{|l|}
$\mathrm{TiCu}_{0.5} \mathrm{Al}_{2.5}$ \\
$\mathrm{Ti}_{1.15} \mathrm{Cu}_{0.09} \mathrm{Al}_{2.76}$ \\
\end{tabular} & $\begin{array}{l}\mathrm{Cu}_{3} \mathrm{Au}, P m-3 m, \mathrm{a}=3.9416(2) \AA \\
\mathrm{ZrAl}_{3}, I 4 / m m m, \mathrm{a}=3.8935(3), \mathrm{c}=16.6423(2) \AA\end{array}$ \\
\hline $\mathrm{Ti}_{25} \mathrm{Cu}_{7.5} \mathrm{Al}_{67.5}$ & $\begin{array}{l}\mathrm{TiCu}_{0.5} \mathrm{Al}_{2.5} \\
\mathrm{Ti}_{1.15} \mathrm{Cu}_{0.09} \mathrm{Al}_{2.76} \\
\end{array}$ & $\begin{array}{l}\mathrm{Cu}_{3} \mathrm{Au}, P m-3 m, \mathrm{a}=3.9384(6) \AA \\
\mathrm{ZrAl}_{3}, I 4 / m m m, \mathrm{a}=3.8903(1), \mathrm{c}=16.625(7) \AA\end{array}$ \\
\hline $\mathrm{Ti}_{25} \mathrm{Cu}_{10} \mathrm{Al}_{65}$ & $\mathrm{TiCu}_{0.5} \mathrm{Al}_{2.5}$ & $\mathrm{Cu}_{3} \mathrm{Au}, P m-3 m, \mathrm{a}=3.9386(6) \AA$ \\
\hline $\mathrm{Ti}_{25} \mathrm{Cu}_{15} \mathrm{Al}_{60}$ & $\mathrm{TiCu}_{0.5} \mathrm{Al}_{2.5}$ & $\mathrm{Cu}_{3} \mathrm{Au}, P m-3 m, \mathrm{a}=3.9193(5) \AA$ \\
\hline $\mathrm{Ti}_{25} \mathrm{Cu}_{50} \mathrm{Al}_{25}$ & $\begin{array}{l}\mathrm{TiCu}_{2} \mathrm{Al} \\
\mathrm{Cu}_{9} \mathrm{Al}_{4} \\
\mathrm{TiCu}_{0.5} \mathrm{Al}_{2.5} \\
\end{array}$ & $\begin{array}{l}\mathrm{MnCu}_{2} \mathrm{Al}, F m-3 m, \mathrm{a}=6.016(1) \AA \\
\mathrm{Cu}_{9} \mathrm{Al}_{4}, P-43 m, \mathrm{a}=8.708(2) \AA \\
\mathrm{Cu}_{3} \mathrm{Au}, P m-3 m, \mathrm{a}=3.907(1) \AA\end{array}$ \\
\hline $\mathrm{Ti}_{25} \mathrm{Cu}_{55} \mathrm{Al}_{20}$ & $\mathrm{TiCu}_{2} \mathrm{Al}$ & $\mathrm{MnCu}_{2} \mathrm{Al}, F m-3 m, \mathrm{a}=6.003(2) \AA$ \\
\hline $\mathrm{Ti}_{25} \mathrm{Cu}_{60} \mathrm{Al}_{15}$ & $\mathrm{TiCu}_{2} \mathrm{Al}$ & $\mathrm{MnCu}_{2} \mathrm{Al}, F m-3 m, \mathrm{a}=5.986(1) \AA$ \\
\hline $\mathrm{Ti}_{25} \mathrm{Cu}_{63} \mathrm{Al}_{12}$ & $\mathrm{TiCu}_{2} \mathrm{Al}$ & $\mathrm{MnCu}_{2} \mathrm{Al}, F m-3 m, \mathrm{a}=5.976(3) \AA$ \\
\hline $\mathrm{Ti}_{27} \mathrm{Cu}_{2} \mathrm{Al}_{71}$ & $\mathrm{Ti}_{1.15} \mathrm{Cu}_{0.09} \mathrm{Al}_{2.76}$ & $\mathrm{ZrAl}_{3}, I 4 / \mathrm{mmm}, \mathrm{a}=3.9159(3), \mathrm{c}=16.556(2) \AA$ \\
\hline $\mathrm{Ti}_{29} \mathrm{Cu}_{2} \mathrm{Al}_{69}$ & $\mathrm{Ti}_{1.15} \mathrm{Cu}_{0.09} \mathrm{Al}_{2.76}$ & $\mathrm{ZrAl}_{3}, I 4 / \mathrm{mmm}, \mathrm{a}=3.9150(1), \mathrm{c}=16.554(7) \AA$ \\
\hline $\mathrm{Ti}_{28} \mathrm{Cu}_{34} \mathrm{Al}_{38}$ & $\mathrm{TiCu}_{2} \mathrm{Al}$ & $\mathrm{MnCu}_{2} \mathrm{Al}, F m-3 m, \mathrm{a}=6.0111(4) \AA$ \\
\hline $\mathrm{Ti}_{30} \mathrm{Cu}_{40} \mathrm{Al}_{30}$ & \begin{tabular}{|l|}
$\mathrm{TiCuAl}$ \\
$\mathrm{TiCu}_{2} \mathrm{Al}$ \\
\end{tabular} & $\begin{array}{l}\mathrm{MgZn}_{2}, P 6_{3} / m m c, \mathrm{a}=5.0303(7), \mathrm{c}=8.098(1) \AA \\
\mathrm{MnCu}_{2} \mathrm{Al}, F m-3 m, \mathrm{a}=6.0212(9) \AA\end{array}$ \\
\hline $\mathrm{Ti}_{30} \mathrm{Cu}_{50} \mathrm{Al}_{20}$ & $\begin{array}{l}\mathrm{TiCu}_{2} \mathrm{Al} \\
\mathrm{Ti}_{2} \mathrm{Cu} \\
\end{array}$ & $\begin{array}{l}\mathrm{MnCu}_{2} \mathrm{Al}, F m-3 m, \mathrm{a}=6.005(1) \AA \\
\mathrm{Zr}_{2} \mathrm{Cu}, I 4 / m m m, \mathrm{a}=2.978(2), \mathrm{c}=10.57(1) \AA\end{array}$ \\
\hline $\mathrm{Ti}_{30} \mathrm{Cu}_{60} \mathrm{Al}_{10}$ & \begin{tabular}{|l|}
$\mathrm{TiCu}_{2} \mathrm{Al}$ \\
$\mathrm{TiCu}$ \\
\end{tabular} & $\begin{array}{l}\mathrm{MnCu}_{2} \mathrm{Al}, F m-3 m, \mathrm{a}=5.971(2) \AA \\
\mathrm{TiCu}, P 4 / m m m, \mathrm{a}=3.137(2), \mathrm{c}=2.847(5) \AA\end{array}$ \\
\hline $\mathrm{Ti}_{31} \mathrm{Cu}_{2} \mathrm{Al}_{67}$ & $\mathrm{Ti}_{1.15} \mathrm{Cu}_{0.09} \mathrm{Al}_{2.76}$ & $\mathrm{ZrAl}_{3}, I 4 / \mathrm{mmm}, \mathrm{a}=3.9155(1), \mathrm{c}=16.559(7) \AA$ \\
\hline $\mathrm{Ti}_{33.3} \mathrm{Cu}_{16} \mathrm{Al}_{50.7}$ & $\begin{array}{l}\mathrm{TiCuAl} \\
\mathrm{TiAl} \\
\mathrm{TiCu}_{0.5} \mathrm{Al}_{2.5} \\
\end{array}$ & $\begin{array}{l}\mathrm{MgZn}_{2}, P 6_{3} / m m c, \mathrm{a}=5.0303(7), \mathrm{c}=8.098(1) \AA \\
\mathrm{CuAu}, P 4 / m m m, \mathrm{a}=2.8117(6), \mathrm{c}=4.050(1) \AA \\
\mathrm{Cu}_{3} \mathrm{Au}, P m-3 m, \mathrm{a}=3.9265(6) \AA\end{array}$ \\
\hline $\mathrm{Ti}_{33.3} \mathrm{Cu}_{18} \mathrm{Al}_{48.7}$ & $\begin{array}{l}\mathrm{TiCuAl} \\
\mathrm{TiCu}_{0.5} \mathrm{Al}_{2.5} \\
\end{array}$ & $\begin{array}{l}\mathrm{MgZn}_{2}, P 6_{3} / m m c, \mathrm{a}=5.0615(5), \mathrm{c}=8.1789(9) \AA \\
\mathrm{Cu}_{3} \mathrm{Au}, P m-3 m, \mathrm{a}=3.9213(5) \AA\end{array}$ \\
\hline $\mathrm{Ti}_{33.3} \mathrm{Cu}_{23} \mathrm{Al}_{43.7}$ & TiCuAl & $\mathrm{MgZn}_{2}, P 6_{3} / m m c, \mathrm{a}=5.0553(4), \mathrm{c}=8.1361(7) \AA$ \\
\hline $\mathrm{Ti}_{33.3} \mathrm{Cu}_{28} \mathrm{Al}_{38.7}$ & TiCuAl & $\mathrm{MgZn}_{2}, P 6_{3} / m m c, \mathrm{a}=5.0364(6), \mathrm{c}=8.1065(9) \AA$ \\
\hline $\mathrm{Ti}_{33.3} \mathrm{Cu}_{33.3} \mathrm{Al}_{33.3}$ & $\begin{array}{l}\mathrm{TiCuAl} \\
\mathrm{TiCu}_{0.5} \mathrm{Al}_{2.5} \\
\end{array}$ & $\begin{array}{l}\mathrm{MgZn}_{2}, P 6_{3} / m m c, \mathrm{a}=5.0165(5), \mathrm{c}=8.0920(1) \AA \\
\mathrm{Cu}_{3} \mathrm{Au}, P m-3 m, \mathrm{a}=3.9101(5) \AA\end{array}$ \\
\hline $\mathrm{Ti}_{33.3} \mathrm{Cu}_{38} \mathrm{Al}_{28.7}$ & TiCuAl & $\mathrm{MgZn}_{2}, P 6_{3} / m m c, \mathrm{a}=5.0053(5), \mathrm{c}=8.0906(8) \AA$ \\
\hline $\mathrm{Ti}_{35} \mathrm{Cu}_{5} \mathrm{Al}_{60}$ & $\begin{array}{l}\mathrm{TiCu}_{0.5} \mathrm{Al}_{2.5} \\
\mathrm{TiAl} \\
\end{array}$ & $\begin{array}{l}\mathrm{Cu}_{3} \mathrm{Au}, P m-3 m, \mathrm{a}=3.928(2) \AA \\
\mathrm{CuAu}, P 4 / m m m, \mathrm{a}=2.815(2), \mathrm{c}=4.045(3) \AA\end{array}$ \\
\hline $\mathrm{Ti}_{40} \mathrm{Cu}_{40} \mathrm{Al}_{20}$ & $\begin{array}{l}\mathrm{TiCu}_{2} \mathrm{Al} \\
\mathrm{TiCu}_{0.5} \mathrm{Al}_{2.5}\end{array}$ & $\begin{array}{l}\mathrm{MnCu}_{2} \mathrm{Al}, P m-3 m, \mathrm{a}=5.993(3) \AA \\
\mathrm{Cu}_{3} \mathrm{Au}, P m-3 m, \mathrm{a}=3.920(2) \AA\end{array}$ \\
\hline
\end{tabular}


Table 1 (continued)

\begin{tabular}{|c|c|c|}
\hline 1 & 2 & 3 \\
\hline $\mathrm{Ti}_{40} \mathrm{Cu}_{50} \mathrm{Al}_{10}$ & $\begin{array}{l}\mathrm{TiCu}_{2} \mathrm{Al} \\
\mathrm{TiCu} \\
\mathrm{Ti}_{2} \mathrm{Cu}\end{array}$ & $\begin{array}{l}\mathrm{MnCu}_{2} \mathrm{Al}, F m-3 m, \mathrm{a}=6.061(5) \AA \\
\mathrm{TiCu}, P 4 / m m m, \mathrm{a}=3.131(3), \mathrm{c}=2.9110(2) \AA \\
\mathrm{Zr}_{2} \mathrm{Cu}, I 4 / m m m, \mathrm{a}=2.999(7), \mathrm{c}=10.69(4) \AA\end{array}$ \\
\hline $\mathrm{Ti}_{45} \mathrm{Cu}_{10} \mathrm{Al}_{45}$ & $\begin{array}{l}\text { TiCuAl } \\
\text { TiAl }\end{array}$ & $\begin{array}{l}\mathrm{MgZn}_{2}, P 6_{3} / m m c, \mathrm{a}=5.034(2), \mathrm{c}=8.107(3) \AA \\
\mathrm{CuAu}, P 4 / m m m, \mathrm{a}=2.820(1), \mathrm{c}=4.049(2) \AA\end{array}$ \\
\hline $\mathrm{Ti}_{45} \mathrm{Cu}_{20} \mathrm{Al}_{35}$ & $\begin{array}{l}\mathrm{TiCuAl} \\
\mathrm{Ti}_{3} \mathrm{Al}\end{array}$ & $\begin{array}{l}\mathrm{MgZn}_{2}, P 6_{3} / m m c, \mathrm{a}=5.026(2), \mathrm{c}=8.104(3) \AA \\
\mathrm{Mg}_{3} \mathrm{Cd}, P 6_{3} / m m c, \mathrm{a}=5.767(3), \mathrm{c}=4.633(2) \AA\end{array}$ \\
\hline $\mathrm{Ti}_{50} \mathrm{Cu}_{40} \mathrm{Al}_{10}$ & $\begin{array}{l}\mathrm{Ti}_{2} \mathrm{Cu} \\
\mathrm{TiCuAl} \\
\mathrm{TiCu}_{2} \mathrm{Al} \\
\end{array}$ & $\begin{array}{l}\mathrm{Zr}_{2} \mathrm{Cu}, I 4 / m m m, \mathrm{a}=3.039(2), \mathrm{c}=10.693(9) \AA \\
\mathrm{MgZn}_{2}, P 6_{3} / m m c, \mathrm{a}=5.013(3), \mathrm{c}=8.179(9) \AA \\
\mathrm{MnCu}_{2} \mathrm{Al}, F m-3 m, \mathrm{a}=6.112(4) \AA\end{array}$ \\
\hline $\mathrm{Ti}_{60} \mathrm{Cu}_{10} \mathrm{Al}_{30}$ & $\begin{array}{l}\mathrm{Ti}_{3} \mathrm{Al} \\
\mathrm{Ti}_{2} \mathrm{Cu}\end{array}$ & $\begin{array}{l}\mathrm{Mg}_{3} \mathrm{Cd}, P 6_{3} / m m c, \mathrm{a}=5.766(6), \mathrm{c}=4.620(3) \AA \\
\mathrm{Zr}_{2} \mathrm{Cu}, I 4 / \mathrm{mm}, \mathrm{a}=2.981(4), \mathrm{c}=10.82(2) \AA\end{array}$ \\
\hline
\end{tabular}

Note: ${ }^{\mathrm{a}}-\mathrm{TiCu}_{0.5} \mathrm{Al}_{2.5}: \mathrm{TiCu}_{\mathrm{x}} \mathrm{Al}_{3-\mathrm{x}}, \mathrm{x}=0.30(3)-0.60(3) ; \mathrm{TiCu}_{2} \mathrm{Al}: \mathrm{TiCu}_{2+\mathrm{x}} \mathrm{Al}_{1-\mathrm{x}}, \mathrm{x}=0-0.52(2)$; $\mathrm{TiCuAl}_{\mathrm{TiCu}} \mathrm{Tl}_{2-\mathrm{x}}, \mathrm{x}=0.54(3)-1.14(3)$.

Table 2

Crystallographic parameters of the binary and ternary compounds observed in the system $\mathrm{Ti}-\mathrm{Cu}-\mathrm{Al}$ at $800^{\circ} \mathrm{C}$

\begin{tabular}{|c|c|c|c|c|c|c|}
\hline Compound & Structure type & Pearson symbol & Space group & $\mathrm{a}, \AA$ & $\mathrm{c}, \AA$ & Reference \\
\hline $\mathrm{TiCu}_{0.12} \mathrm{Al}_{2.88}$ & \multirow[b]{2}{*}{$\mathrm{Cu}_{3} \mathrm{Au}$} & \multirow[b]{2}{*}{$c P 4$} & \multirow[b]{2}{*}{$P m-3 m$} & 3.927 & - & [4] \\
\hline $\begin{array}{l}\mathrm{TiCu}_{0.30(3)} \mathrm{Al}_{2.70(3)^{-}} \\
\mathrm{TiCu}_{0.60(3)} \mathrm{Al}_{2.40(3)}\end{array}$ & & & & $\begin{array}{c}3.9384(6)- \\
3.9193(5)\end{array}$ & - & a \\
\hline $\mathrm{TiCu}_{0.16} \mathrm{Al}_{2.84}$ & \multirow{2}{*}{$\mathrm{ZrAl}_{3}$} & \multirow{2}{*}{$t I 16$} & \multirow{2}{*}{$I 4 / \mathrm{mmm}$} & 3.901 & 16.60 & [4] \\
\hline $\mathrm{Ti}_{1.15(2)} \mathrm{Cu}_{0.09(1)} \mathrm{Al}_{2.76(3)}$ & & & & $3.9159(3)$ & $16.556(2)$ & $\mathrm{a}$ \\
\hline $\mathrm{TiCu}_{2.25} \mathrm{Al}_{0.75}$ & \multirow[b]{2}{*}{$\mathrm{MnCu}_{2} \mathrm{Al}$} & \multirow[b]{2}{*}{$c F 16$} & \multirow[b]{2}{*}{$F m-3 m$} & 5.93 & - & {$[4]$} \\
\hline $\begin{array}{l}\mathrm{TiCu}_{2} \mathrm{Al}- \\
\mathrm{TiCu}_{2.52(2)} \mathrm{Al}_{0.48(2)}\end{array}$ & & & & $\begin{array}{c}6.016(1)- \\
5.976(3)\end{array}$ & - & $\mathrm{a}$ \\
\hline TiCuAl & \multirow[b]{2}{*}{$\mathrm{MgZn}_{2}$} & \multirow[b]{2}{*}{$h P 12$} & \multirow[b]{2}{*}{$\mathrm{Pb}_{3} / m m c$} & 5.00 & 8.165 & {$[4]$} \\
\hline $\begin{array}{l}\mathrm{TiCu}_{0.54(3)} \mathrm{Al}_{1.46(3)^{-}} \\
\mathrm{TiCu}_{1.14(3)} \mathrm{Al}_{0.86(3)}\end{array}$ & & & & $\begin{array}{c}5.0615(5)- \\
5.0053(5)\end{array}$ & $\begin{array}{c}8.1789(9)- \\
8.0906(8)\end{array}$ & a \\
\hline \multirow{2}{*}{$\mathrm{TiCu}$} & \multirow{2}{*}{$\mathrm{TiCu}$} & \multirow{2}{*}{$t P 2$} & \multirow{2}{*}{$P 4 / \mathrm{mmm}$} & 3.140 & 2.856 & {$[2]$} \\
\hline & & & & $3.131(3)$ & $2.9110(2)$ & ${ }^{a}$ \\
\hline \multirow{2}{*}{$\mathrm{Ti}_{2} \mathrm{Cu}$} & \multirow{2}{*}{$\mathrm{Zr}_{2} \mathrm{Cu}$} & \multirow{2}{*}{$t I 6$} & \multirow{2}{*}{$\mathrm{I} / \mathrm{mmm}$} & 2.935 & 10.772 & [9] \\
\hline & & & & $3.039(2)$ & $10.693(9)$ & $\mathrm{a}$ \\
\hline \multirow{2}{*}{ TiAl } & \multirow{2}{*}{$\mathrm{CuAu}$} & \multirow{2}{*}{$t P 2$} & \multirow{2}{*}{$P 4 / \mathrm{mmm}$} & 2.8284 & 4.075 & {$[10]$} \\
\hline & & & & $2.815(2)$ & $4.045(3)$ & $\mathrm{a}$ \\
\hline \multirow{2}{*}{$\mathrm{Ti}_{3} \mathrm{Al}$} & \multirow{2}{*}{$\mathrm{Mg}_{3} \mathrm{Cd}$} & \multirow{2}{*}{$h P 8$} & \multirow{2}{*}{$\mathrm{Pb}_{3} / m m c$} & 5.764 & 4.664 & [11] \\
\hline & & & & $5.766(6)$ & $4.620(3)$ & $\mathrm{a}$ \\
\hline \multirow{2}{*}{$\mathrm{Cu}_{1.7} \mathrm{Al}$} & \multirow{2}{*}{$\mathrm{Co}_{1.75} \mathrm{Ge}$} & \multirow{2}{*}{$h P 6$} & \multirow{2}{*}{$P 6_{3} / m m c$} & 4.146 & 5.063 & {$[2]$} \\
\hline & & & & $4.108(1)$ & $5.014(1)$ & $\mathrm{a}$ \\
\hline \multirow{2}{*}{$\mathrm{Cu}_{9} \mathrm{Al}_{4}$} & \multirow{2}{*}{$\mathrm{Cu}_{9} \mathrm{Al}_{4}$} & \multirow{2}{*}{$c P 52$} & $P-43 m$ & 8.707 & 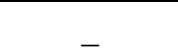 & [2] \\
\hline & & & $P-45 m$ & $8.694(1)$ & - & $\mathrm{a}$ \\
\hline
\end{tabular}

Note: ${ }^{a}-$ this work.

$\mathrm{TiCu}_{\mathrm{x}} \mathrm{Al}_{3-\mathrm{x}}, \quad \mathrm{x}=0.30(3)-0.60(3), \mathrm{TiCu}_{2+\mathrm{x}} \mathrm{Al}_{1-\mathrm{x}}$, $\mathrm{x}=0-0.52(2)$, and $\left.\mathrm{TiCu}_{\mathrm{x}} \mathrm{Al}_{2-\mathrm{x}}, \mathrm{x}=0.54(3)-1.14(3)\right)$ were determined and are listed in Table 2. It should be mentioned that the high-temperature phase with W-type structure ( $\beta$ phase), reported to form at 70-
80 at.\% $\mathrm{Cu}$ above $567^{\circ} \mathrm{C}$ in the $\mathrm{Cu}-\mathrm{Al}$ system, was not observed in this work, presumably because of not efficient quenching.

Three ternary compounds with variable composition at $800^{\circ} \mathrm{C}$ exhibit the following 
Table 3

Unit-cell parameters of ternary compounds with extended homogeneity ranges in the system $\mathrm{Ti}-\mathrm{Cu}-\mathrm{Al}$ at $\mathrm{800}^{\circ} \mathrm{C}$

\begin{tabular}{|c|c|c|c|c|}
\hline Nominal composition of the sample & $\mathrm{x}$ & $\mathrm{a}, \AA$ & $\mathrm{c}, \AA$ & $\mathrm{V}, \AA^{3}$ \\
\hline \multicolumn{5}{|c|}{$\mathrm{TiCu}_{\mathrm{x}} \mathrm{Al}_{3-\mathrm{x}}, \mathrm{x}=0.30(3)-0.60(3)$ (structure type $\left.\mathrm{Cu}_{3} \mathrm{Au}\right)$} \\
\hline $\mathrm{Ti}_{25} \mathrm{Cu}_{7.5} \mathrm{Al}_{67.5}$ & 0.30 & $3.9384(6)$ & - & $61.09(2)$ \\
\hline $\mathrm{Ti}_{25} \mathrm{Cu}_{10} \mathrm{Al}_{65}$ & 0.40 & $3.9386(6)$ & - & $61.10(2)$ \\
\hline $\mathrm{Ti}_{25} \mathrm{Cu}_{15} \mathrm{Al}_{60}$ & 0.60 & $3.9193(5)$ & - & $60.20(1)$ \\
\hline \multicolumn{5}{|c|}{$\mathrm{TiCu}_{2+\mathrm{x}} \mathrm{Al}_{1-\mathrm{x}}, \mathrm{x}=0-0.52(2)$ (structure type $\mathrm{MnCu}_{2} \mathrm{Al}$ ) } \\
\hline $\mathrm{Ti}_{25} \mathrm{Cu}_{50} \mathrm{Al}_{25}$ & 0 & $6.016(1)$ & - & $217.7(1)$ \\
\hline $\mathrm{Ti}_{25} \mathrm{Cu}_{55} \mathrm{Al}_{20}$ & 0.20 & $6.003(2)$ & - & $216.3(1)$ \\
\hline $\mathrm{Ti}_{25} \mathrm{Cu}_{60} \mathrm{Al}_{15}$ & 0.40 & $5.986(1)$ & - & $214.5(1)$ \\
\hline $\mathrm{Ti}_{25} \mathrm{Cu}_{63} \mathrm{Al}_{12}$ & 0.52 & $5.976(3)$ & - & $213.4(2)$ \\
\hline \multicolumn{5}{|c|}{$\mathrm{TiCu}_{\mathrm{x}} \mathrm{Al}_{2-\mathrm{x}}, \mathrm{x}=0.54(3)-1.14(3)$ (structure type $\mathrm{MgZn}_{2}$ ) } \\
\hline $\mathrm{Ti}_{33.3} \mathrm{Cu}_{18} \mathrm{Al}_{48.7}$ & 0.54 & $5.0615(5)$ & $8.1789(9)$ & $181.4(3)$ \\
\hline $\mathrm{Ti}_{33.3} \mathrm{Cu}_{23} \mathrm{Al}_{43.7}$ & 0.69 & $5.0553(4)$ & $8.1361(7)$ & $180.1(3)$ \\
\hline $\mathrm{Ti}_{33.3} \mathrm{Cu}_{28} \mathrm{Al}_{38.7}$ & 0.84 & $5.0364(6)$ & $8.1065(9)$ & $178.1(4)$ \\
\hline $\mathrm{Ti}_{33.3} \mathrm{Cu}_{33.3} \mathrm{Al}_{33.3}$ & 1 & $5.0165(5)$ & $8.0920(1)$ & $176.4(3)$ \\
\hline $\mathrm{Ti}_{33.3} \mathrm{Cu}_{38} \mathrm{Al}_{28.7}$ & 1.14 & $5.0053(5)$ & $8.0906(8)$ & $175.5(3)$ \\
\hline
\end{tabular}

homogeneity ranges: 7.5 at.\% $\mathrm{Al}(\mathrm{Cu})$ along the isoconcentrate 25 at.\% $\mathrm{Ti}\left(\mathrm{TiCu}_{\mathrm{x}} \mathrm{Al}_{3-\mathrm{x}}, \mathrm{x}=0.30(3)-\right.$ $0.60(3)), 13$ at.\% $\mathrm{Al}(\mathrm{Cu})$ along 25 at.\% $\mathrm{Ti}$ $\left(\mathrm{TiCu}_{2+\mathrm{x}} \mathrm{Al}_{1-\mathrm{x}}, \mathrm{x}=0-0.52(2)\right)$, and 20 at. $\% \mathrm{Al}(\mathrm{Cu})$ along 33.3 at.\% $\mathrm{Ti}\left(\mathrm{TiCu}_{\mathrm{x}} \mathrm{Al}_{2-\mathrm{x}}, \mathrm{x}=0.54(3)-1.14(3)\right)$. Table 3 shows the unit-cell parameters as a function of the $\mathrm{Cu}$ content. In the three cases the unit-cell parameters decrease with increasing $\mathrm{Cu}$ content and decreasing $\mathrm{Al}$ content, in agreement with the difference between the atomic radii of $\mathrm{Al}\left(\mathrm{r}_{\mathrm{Al}}=1.43 \AA\right)$ and $\mathrm{Cu}\left(\mathrm{r}_{\mathrm{Cu}}=1.28 \AA\right)$.

\section{Microstructure analysis}

Figures 4-6 show the microstructure of three investigated samples. The compositions of the phases

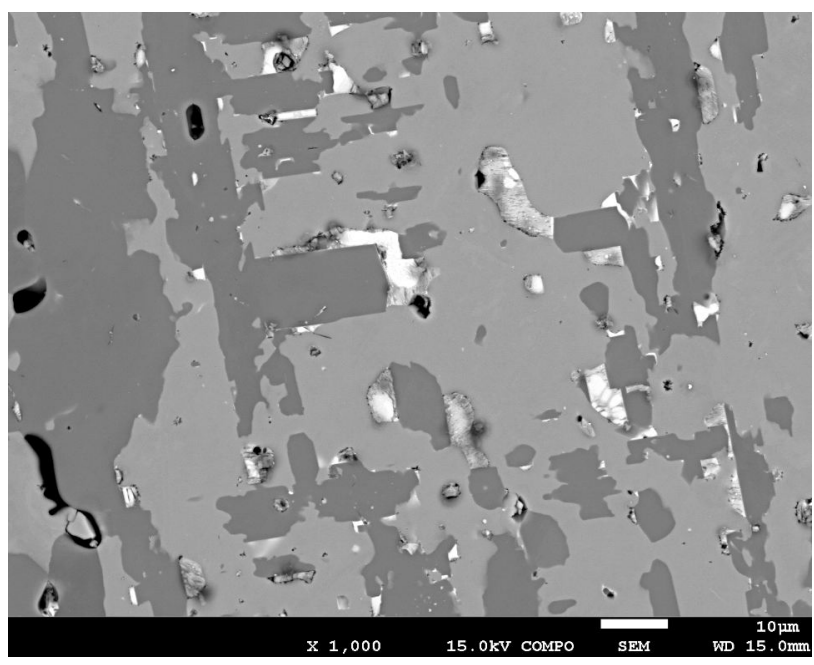

Fig. 4. EDX maps of the sample $\mathrm{Ti}_{25} \mathrm{Cu}_{6.25} \mathrm{Al}_{68.75}$ (grey phase $\mathrm{TiCu}_{0.45(4)} \mathrm{Al}_{2.55(4)}$, dark phase $\left.-\mathrm{Ti}_{1.15(2)} \mathrm{Cu}_{0.09(1)} \mathrm{Al}_{2.76(3)}\right)$ that exist in these samples are given in Table 4. It appears that the Ti content is in general slightly lower than expected (approx. 5 at.\%) and the Ti content in the proposed chemical formulas was normalized to 25 at.\% for the $\mathrm{Cu}_{3} \mathrm{Au}$-type and Heusler phases and 33.3 at.\% for the Laves phase. According to the $\mathrm{X}$-ray diffraction diagram, the sample $\mathrm{Ti}_{25} \mathrm{Cu}_{6.25} \mathrm{Al}_{68.75}$ contains two phases. The composition of the majority phase (grey phase in Fig. 4) with $\mathrm{Cu}_{3} \mathrm{Au}$-type structure was found to be $\mathrm{Ti}_{23.8} \mathrm{Cu}_{11.5} \mathrm{Al}_{64.7}$ from EDX analysis, normalized to $\mathrm{TiCu}_{0.45(4)} \mathrm{Al}_{2.55(4)}$, which is well within the homogeneity range determined by X-ray diffraction (Table 3). The dark phase with $\mathrm{ZrAl}_{3}-$ type structure (see below) was found to contain slightly more than 25 at.\% $\mathrm{Ti}$, in agreement with earlier reports. Assuming the same underestimation of the Ti content as in the other cases, we propose the approximate composition $\mathrm{Ti}_{1.06} \mathrm{Cu}_{0.08} \mathrm{Al}_{2.86}$.

Table 4

EDX analysis of three samples in the $\mathrm{Ti}-\mathrm{Cu}-\mathrm{Al}$ system

\begin{tabular}{c|c|c}
\hline $\begin{array}{c}\text { Nominal } \\
\text { composition of } \\
\text { the sample }\end{array}$ & $\begin{array}{c}\text { Composition from } \\
\text { EDX, at.\% }\end{array}$ & $\begin{array}{c}\text { Proposed chemical } \\
\text { formula }\end{array}$ \\
\hline $\mathrm{Ti}_{25} \mathrm{Cu}_{6.25} \mathrm{Al}_{68.75}$ & $\mathrm{Ti}_{23.81} \mathrm{Cu}_{11.45} \mathrm{Al}_{64.74}$ & $\mathrm{TiCu}_{0.45} \mathrm{Al}_{2.55}$ \\
$\mathrm{Ti}_{25.25} \mathrm{Cu}_{1.90} \mathrm{Al}_{72.85}$ & $\mathrm{Ti}_{1.15} \mathrm{Cu}_{0.09} \mathrm{Al}_{2.76}$ \\
\hline $\mathrm{Ti}_{25} \mathrm{Cu}_{50} \mathrm{Al}_{25}{ }^{\mathrm{a}}$ & $\mathrm{Ti}_{23.67} \mathrm{Cu}_{53.65} \mathrm{Al}_{22.68}$ & $\mathrm{TiCu}_{2.11} \mathrm{Al}_{0.89}$ \\
\hline $\mathrm{Ti}_{33.3} \mathrm{Cu}_{23} \mathrm{Al}_{43.7}$ & $\mathrm{Ti}_{31.68} \mathrm{Cu}_{24.69} \mathrm{Al}_{43.63}$ & $\mathrm{TiCu}_{0.72} \mathrm{Al}_{1.28}$ \\
$\mathrm{Ti}_{46.63} \mathrm{Al}_{53.37}$ & $\mathrm{Ti}_{0.93} \mathrm{Al}_{1.07}$ \\
\hline
\end{tabular}

Note: a ${ }^{a}$ the sample contains small amounts of $\mathrm{Cu}_{9} \mathrm{Al}_{4}$ and $\mathrm{TiCu}_{0.5} \mathrm{Al}_{2.5}$.

The dominating phase in the $\mathrm{Ti}_{25} \mathrm{Cu}_{50} \mathrm{Al}_{25}$ 
sample (Fig. 5) is the Heusler compound with $\mathrm{MnCu}_{2} \mathrm{Al}$-type structure, accompanied by trace amounts of the ternary $\mathrm{Cu}_{3} \mathrm{Au}$-type phase and binary gamma-brass $\mathrm{Cu}_{9} \mathrm{Al}_{4}$. The chemical formula of the $\mathrm{Cu}_{3} \mathrm{Au}$-type phase was normalized to $\mathrm{TiCu}_{2.11(4)} \mathrm{Al}_{0.89(4)}$. Here too the composition from EDX analysis is in good agreement with the homogeneity range from X-ray diffraction in Table 3.

The photograph of the sample $\mathrm{Ti}_{33.3} \mathrm{Cu}_{23} \mathrm{Al}_{43.7}$ in Fig. 6 shows two phases. The light phase is the Laves phase with $\mathrm{MgZn}_{2}$-type structure. The normalized composition from the EDX analysis is $\mathrm{TiCu}_{0.72(3)} \mathrm{Al}_{1.28(3)}$, i.e. well inside the homogeneity range $\mathrm{TiCu}_{\mathrm{x}} \mathrm{Al}_{2-\mathrm{x}}, \mathrm{x}=0.54(3)-1.14(3)$. The second phase has the composition $\mathrm{Ti}_{0.93(2)} \mathrm{Al}_{1.07(2)}$ and $\mathrm{CuAu}-$ type structure.

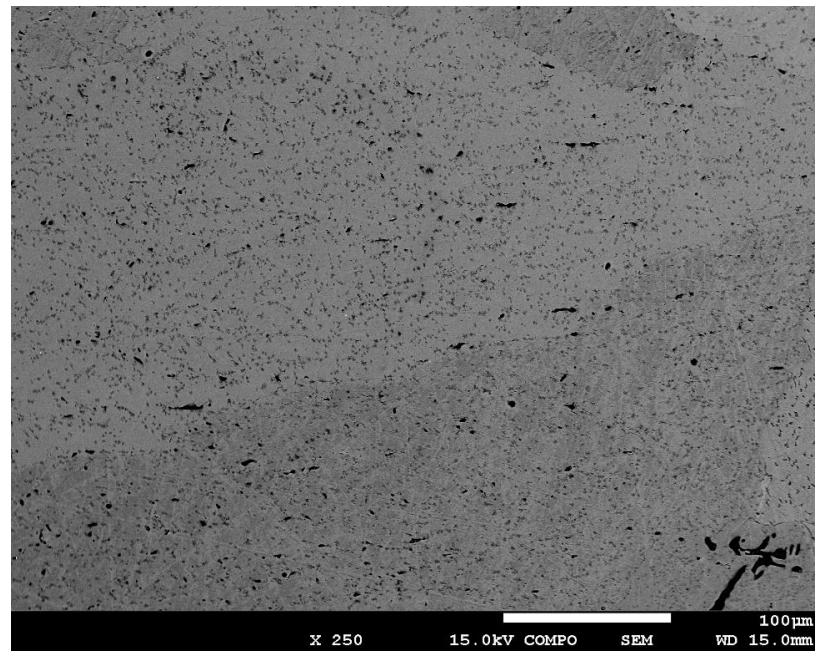

Fig. 5. EDX maps of the sample $\mathrm{Ti}_{25} \mathrm{Cu}_{50} \mathrm{Al}_{25}$ (grey phase $\left.\mathrm{TiCu}_{2.11(4)} \mathrm{Al}_{0.89(4)}\right)$

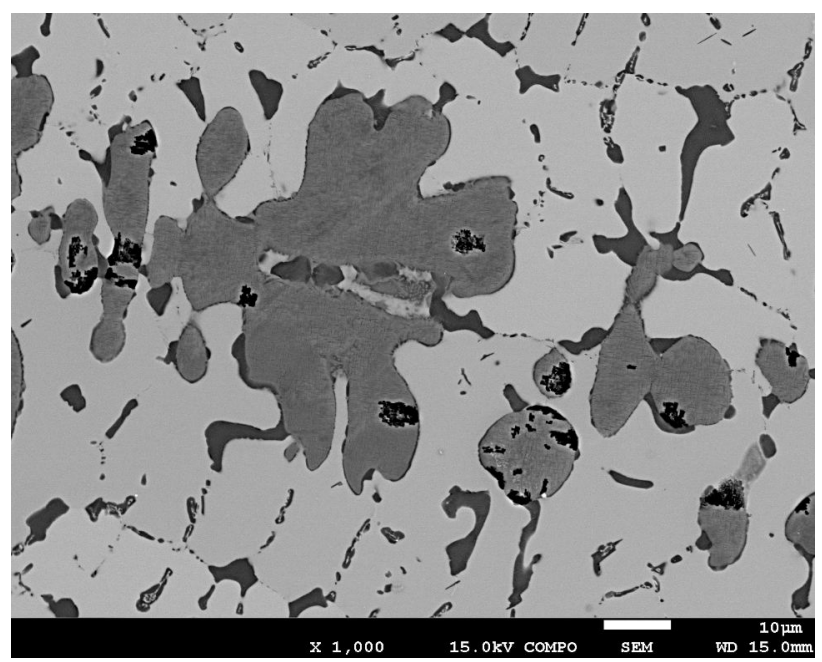

Fig. 6. EDX maps of the sample $\mathrm{Ti}_{33.3} \mathrm{Cu}_{23} \mathrm{Al}_{43.7}$ (light phase $\mathrm{TiCu}_{0.72(3)} \mathrm{Al}_{1.28(3)}$, dark phase $\left.-\mathrm{Ti}_{0.93(2)} \mathrm{Al}_{1.07(2)}\right)$

\section{Differential thermal analysis}

The DTA curve of the sample $\mathrm{Ti}_{25} \mathrm{Cu}_{6.25} \mathrm{Al}_{68.75}$ (Fig. 7,a) shows two thermal effects, at $941.5^{\circ} \mathrm{C} /$ $1326.1^{\circ} \mathrm{C}$ and $936.7^{\circ} \mathrm{C} / 1354.2^{\circ} \mathrm{C}$ in the heating and cooling regimes, respectively. The thermal effects recorded during heating of the sample correspond to the melting temperature of the two ternary compounds: $\mathrm{Ti}_{1.15(2)} \mathrm{Cu}_{0.09(1)} \mathrm{Al}_{2.76(3)}\left(\mathrm{ZrAl}_{3}\right.$-type structure) and $\mathrm{TiCu}_{0.45(4)} \mathrm{Al}_{2.55(4)}\left(\mathrm{Cu}_{3} \mathrm{Au}\right)$. The peaks on the cooling curve show the temperatures of crystallization of these compounds. According to ref. [12], the ternary compound $\mathrm{Ti}_{2} \mathrm{CuAl}_{5}$ forms at $1350^{\circ} \mathrm{C}$, in agreement with our DTA results.

The DTA curve for the sample $\mathrm{Ti}_{25} \mathrm{Cu}_{50} \mathrm{Al}_{25}$ (Fig. 7,b) contains several peaks. On the heating curve, the first one at $953.7^{\circ} \mathrm{C}$ is in agreement with the temperature of peritectoid formation of the roomtemperature modification of the binary compound $\mathrm{Cu}_{9} \mathrm{Al}_{4}\left(873^{\circ} \mathrm{C}\right.$ in ref. [13]). The peak at $1012^{\circ} \mathrm{C}$ corresponds to the structural transition $\mathrm{Cu}_{9} \mathrm{Al}_{4} \mathrm{rt}-$ $\mathrm{Cu}_{9} \mathrm{Al}_{4}$ ht $\left(1022^{\circ} \mathrm{C}\right.$ in ref. [13]), whereas the third peak, at $1067^{\circ} \mathrm{C}$, shows the melting temperature of the ternary compound $\mathrm{TiCu}_{2.11(4)} \mathrm{Al}_{0.89(4)}$ with $\mathrm{MnCu}_{2} \mathrm{Al}$-type structure, in slight disagreement with ref. [12] $\left(1125^{\circ} \mathrm{C}\right)$.

The DTA curve of the sample $\mathrm{Ti}_{33.3} \mathrm{Cu}_{23} \mathrm{Al}_{43.7}$ contains two peaks (Fig. 7,c): the thermal effect on the heating curve at $1240.1^{\circ} \mathrm{C}$ corresponds to the melting temperature of the ternary compound $\mathrm{TiCu}_{0.72(3)} \mathrm{Al}_{1.28(3)}\left(\mathrm{MgZn}_{2}\right)$ and the peak on the cooling curve at $1241.8^{\circ} \mathrm{C}$ to the crystallization of this compound. The results obtained here are in agreement with refs. [5,6] $\left(1200^{\circ} \mathrm{C}\right)$. $800^{\circ} \mathrm{C}$

Isothermal section of the system $\mathrm{Ti}-\mathrm{Cu}-\mathrm{Al}$ at

The isothermal section of the system $\mathrm{Ti}-\mathrm{Cu}-$ $\mathrm{Al}$ at $800^{\circ} \mathrm{C}$ presented in Fig. 8 was constructed based on literature data available for the binary boundary systems and own ternary alloys. Aluminum being liquid at $800^{\circ} \mathrm{C}$, the equilibria in the $\mathrm{Al}$-rich corner are tentative, and those of the $\mathrm{Cu}$-rich corner were assigned in agreement with earlier study [5]. The section contains 18 single-phase regions, 35 twophase and 19 three-phase regions.

According to ref. [13], the solid solubility of $\mathrm{Cu}$ in $\mathrm{Ti}$ is limited to 1.5 at. $\% \mathrm{Cu}$ at $800^{\circ} \mathrm{C}$, while the solubility of $\mathrm{Ti}$ in $\mathrm{Cu}$ exceeds 5 at.\% $\mathrm{Ti}$. $\mathrm{Al}$ dissolves very little $\mathrm{Ti}$, but $\mathrm{Cu}$ dissolves more than 5 at.\% Ti. The solid solubility of $\mathrm{Al}$ in $\mathrm{Cu}$ is almost 18 at.\% Al. Based on the cell parameters of the binary compounds in ternary samples, we deduce that the binary compounds of the systems $\mathrm{Ti}-\mathrm{Cu}$ and $\mathrm{Ti}-\mathrm{Al}$ dissolve less than 2 at.\% of the third component.

As shown above, the existence of three purely 


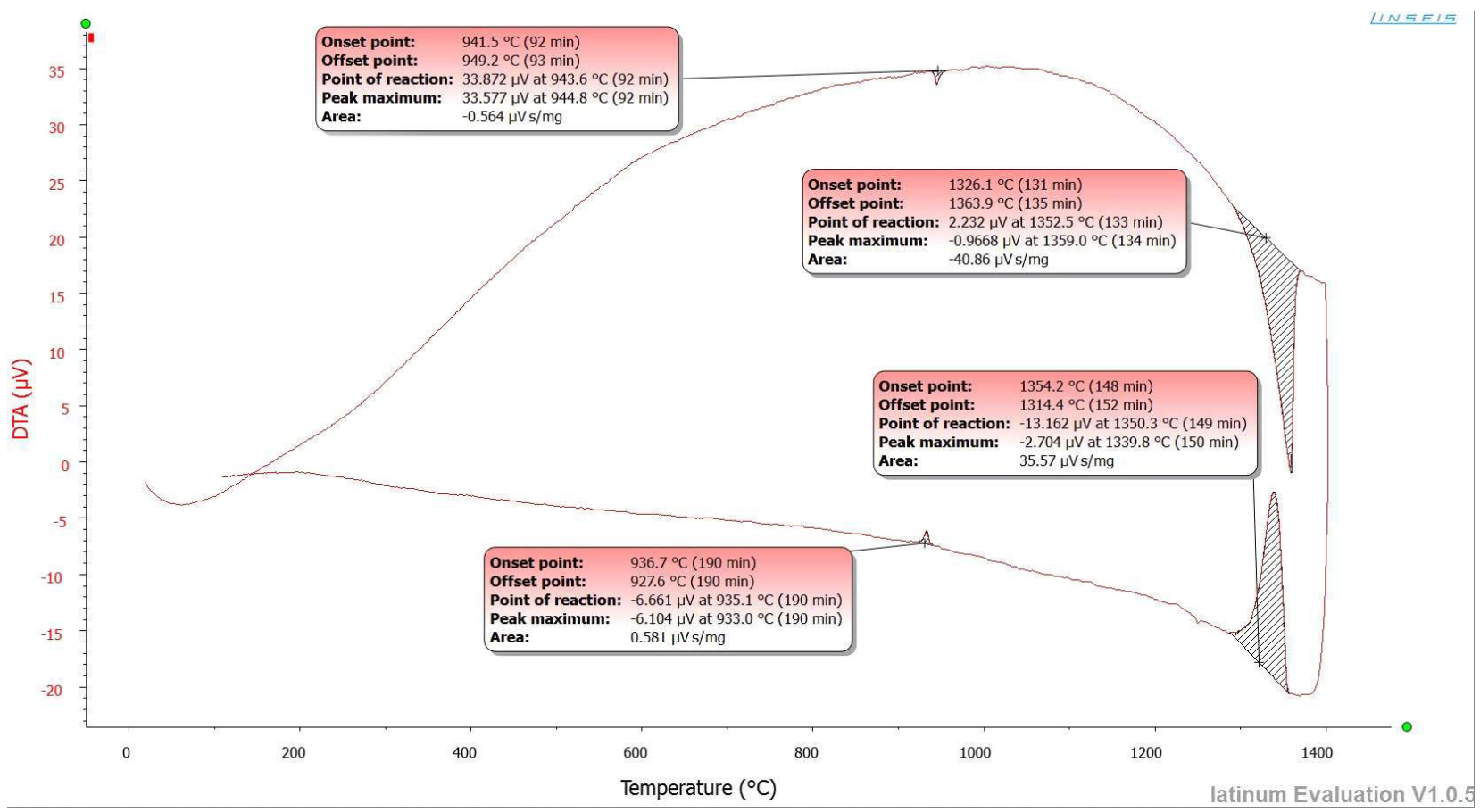

Fig. 7 ,a

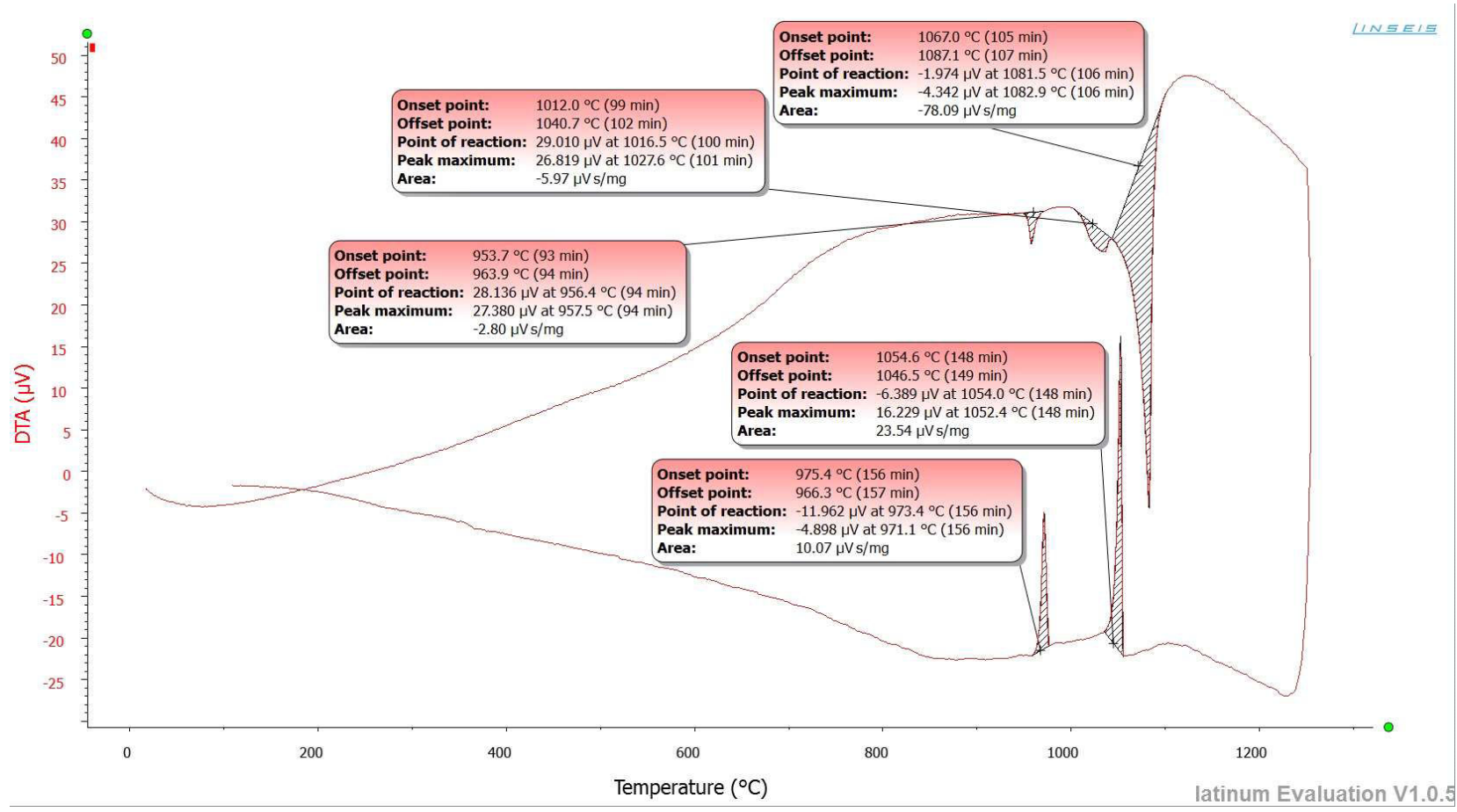

Fig. $7, b$ 


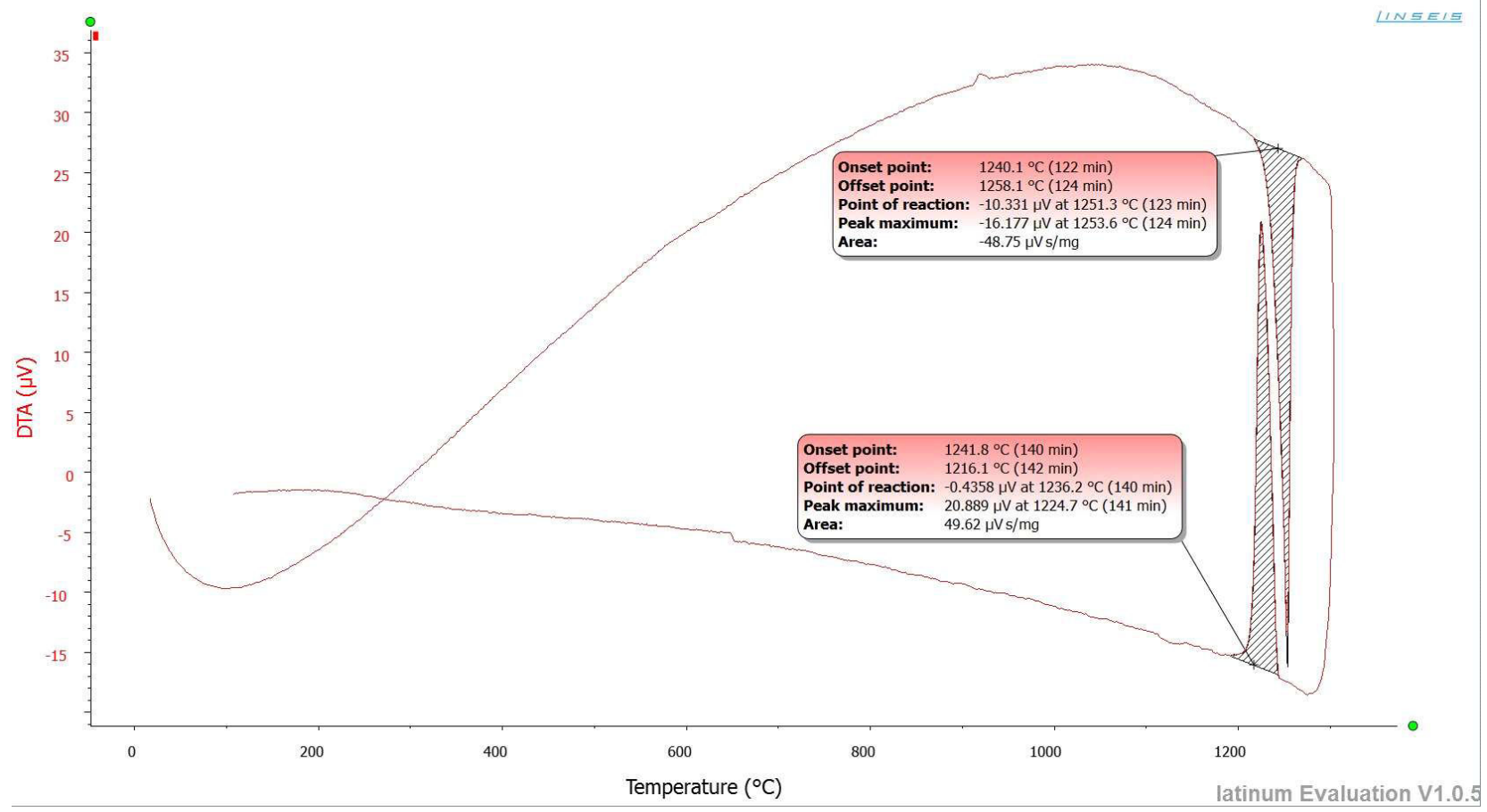

Fig. 7. DTA curves for the samples a $-\mathrm{Ti}_{25} \mathrm{Cu}_{6.25} \mathrm{Al}_{68.75}, \mathrm{~b}-\mathrm{Ti}_{25} \mathrm{Cu}_{50} \mathrm{Al}_{25}$ and $\mathrm{c}-\mathrm{Ti}_{33.3} \mathrm{Cu}_{23} \mathrm{Al}_{43.7}$

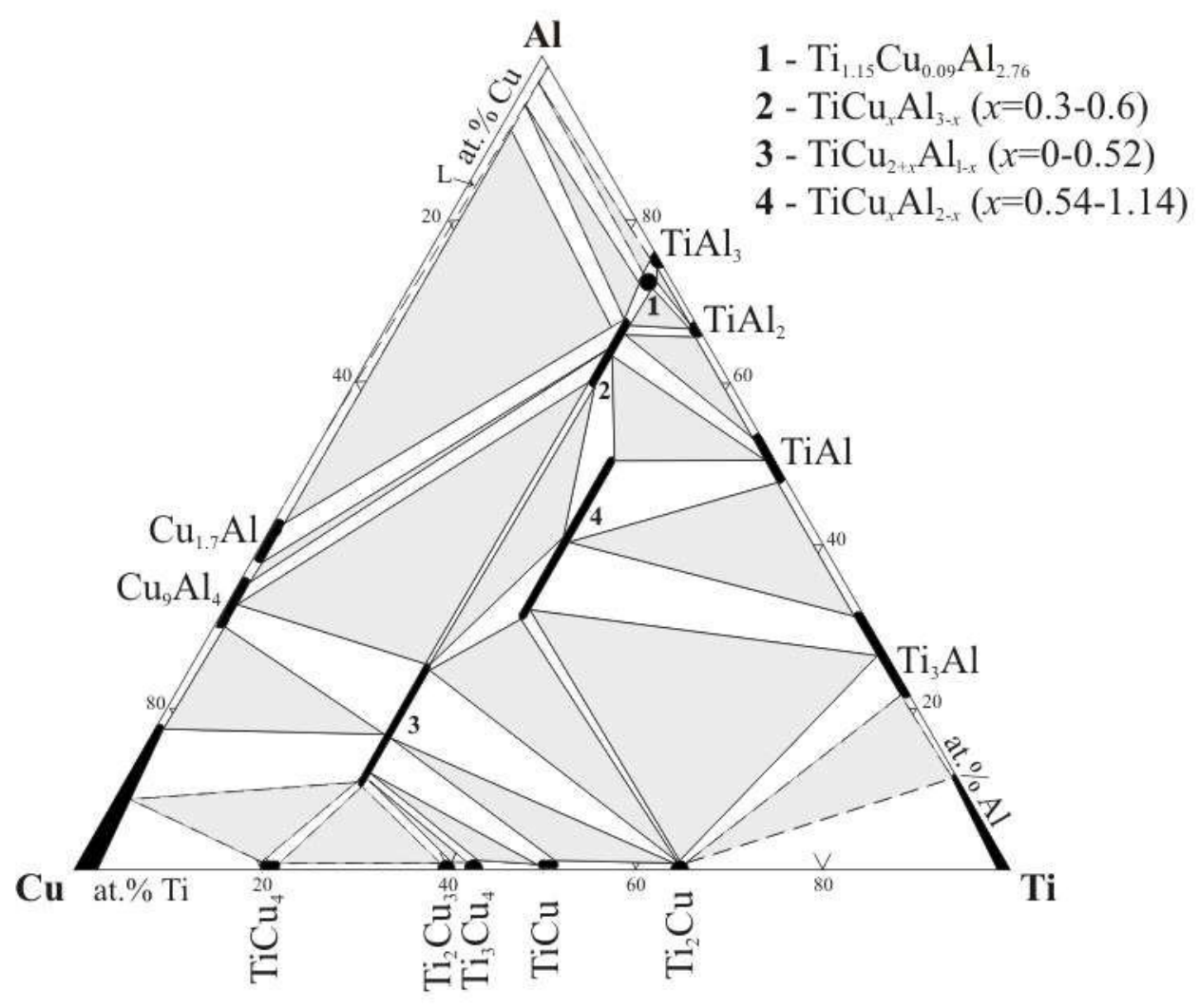

Fig. 8. Isothermal section of the phase diagram of the system $\mathrm{Ti}-\mathrm{Cu}-\mathrm{Al}$ at $800^{\circ} \mathrm{C}$ 
ternary compounds in the system $\mathrm{Ti}-\mathrm{Cu}-\mathrm{Al}$ with large homogeneity ranges was confirmed $(7.5,13$ and 20 at. $\% \mathrm{Al}$ or $\mathrm{Cu}$ along the isoconcentrates 25 at.\% Ti for the former two and 33.3 at.\% for the last one). The additional compound with structure type $\mathrm{ZrAl}_{3}$ is in equilibrium with $\mathrm{TiCu}_{\mathrm{x}} \mathrm{Al}_{3-\mathrm{x}}$, $\mathrm{x}=0.30(3)-0.60(3)$ (structure type $\mathrm{Cu}_{3} \mathrm{Au}$ ) (Fig. 4), which in turn forms equilibria with one binary $\left(\mathrm{Cu}_{9} \mathrm{Al}_{4}\right)$ and two ternary compounds $\left(\mathrm{TiCu}_{2+x} \mathrm{Al}_{1-\mathrm{x}}\right.$, $\mathrm{x}=0-0.52(2)$ (structure type $\mathrm{MnCu}_{2} \mathrm{Al}$ ) and $\mathrm{TiCu}_{\mathrm{x}} \mathrm{Al}_{2-\mathrm{x}}, \mathrm{x}=0.54(3)-1.14(3) \quad$ (structure type $\left.\mathrm{MgZn}_{2}\right)$. The compounds $\mathrm{TiCu}_{2+x} \mathrm{Al}_{1-x}$ and $\mathrm{TiCu}_{\mathrm{x}} \mathrm{Al}_{2-\mathrm{x}}$ are in equilibrium with each other; in addition the former is in equilibrium with $\mathrm{TiCu}$, $\mathrm{Ti}_{2} \mathrm{Cu}$, and $\mathrm{Cu}_{9} \mathrm{Al}_{4}$, whereas the latter forms equilibria with $\mathrm{Ti}_{2} \mathrm{Cu}$, TiAl, and $\mathrm{Ti}_{3} \mathrm{Al}$.

The isothermal section of the phase diagram of the system $\mathrm{Ti}-\mathrm{Cu}-\mathrm{Al}$ at $800^{\circ} \mathrm{C}$ proposed by Markiv et al. [5] does not contain any compound with structure type $\mathrm{ZrAl}_{3}$ (Fig. 3). However, Schubert et al. [4] observed a compound with this structure type at the composition $\mathrm{TiCu}_{0.16} \mathrm{Al}_{2.84}$. They assume that this apparently ternary compound is in fact part of the solid solution of the high-temperature phase reported in the binary $\mathrm{Ti}-\mathrm{Al}$ system at the approximate composition $\mathrm{Ti}_{5} \mathrm{Al}_{11}$. This opinion is also supported by Ran and Stadelmaier [1] in their review of the $\mathrm{Ti}-\mathrm{Cu}-\mathrm{Al}$ system. The structure type $\mathrm{Zr}_{3} \mathrm{Al}$ adopted by the Ti-rich high-temperature phase was confirmed by Bulanova et al. [14] during an investigation of the $\mathrm{Ti}-\mathrm{Al}-\mathrm{Si}$ system, where this phase was also found to incorporate small amounts of $\mathrm{Si}$.

Concerning the homogeneity ranges of the ternary phases, a certain variation of the Ti content cannot be excluded, but the main extension is clearly along the isoconcentrate 25 at.\% $\mathrm{Ti}$ for the cubic close-packed and the Heusler phases, and along 33 at.\% Ti for the Laves phase. In this aspect, the isothermal section constructed here is closer to those determined by Markiv et al. than to that proposed by Virlis and Zwicker. However, in the present investigation the $\mathrm{Cu}$-rich boundary of the Laves phase was found to form equilibrium with $\mathrm{Ti}_{2} \mathrm{Cu}$. Such equilibrium is present in the section at $540^{\circ} \mathrm{C}$ drawn by Virlis and Zwicker, but not in those published by Markiv et al., where, instead, the Heusler phase forms equilibrium with $\mathrm{Ti}_{3} \mathrm{Al}$.

Crystal structure of $\mathrm{Ti}_{1.15} \mathrm{Cu}_{0.09} \mathrm{Al}_{2.76}$

The crystal structure of the ternary aluminide discovered near the composition $\mathrm{TiAl}_{3}$ was solved by direct methods in space group $I 4 / \mathrm{mmm}$ [15] on $\mathrm{X}$-ray powder diffraction data of the sample $\mathrm{Ti}_{27} \mathrm{Cu}_{2} \mathrm{Al}_{71}$. In agreement with the findings by
Schubert et al. [4] it crystallizes in the $\mathrm{ZrAl}_{3}$ structure type (Pearson symbol tII26, space group $14 / \mathrm{mmm}$, $a=3.9159(3), c=16.556(2) \AA)$. Experimental details of the structure refinement and crystallographic data are given in Table 5. Atomic coordinates and isotropic displacement parameters are given in Table 6. The tetragonal unit cell of the $\mathrm{ZrAl}_{3}$ type contains 16 atoms distributed over four sites, one occupied by $\mathrm{Zr}$ and the other three by Al. For the refinement, the $\mathrm{Zr}$ site was assumed to be occupied by Ti atoms, and the other sites by mixtures of $\mathrm{Al}, \mathrm{Ti}$ and $\mathrm{Cu}$. The site in Wyckoff position $4 \mathrm{~d}$ was found to be occupied exclusively by $\mathrm{Al}$ atoms. The final atom coordinates and site occupancies are listed in Table 6.

Table 5

Details of the Rietveld refinement of the sample $\mathbf{T i}_{27} \mathrm{Cu}_{2} \mathrm{Al}_{71}$

\begin{tabular}{l|c}
\hline Composition & $\mathrm{Ti}_{1.15(2)} \mathrm{Cu}_{0.09(1)} \mathrm{Al}_{2.76(3)}$ \\
\hline Structure type & $\mathrm{ZrAl}_{3}$ \\
\hline Space group & \multicolumn{1}{c|}{$\mathrm{c}, \AA / \mathrm{mmm}$} \\
\hline Cell parameters: a, $\AA$ & $3.9159(3)$ \\
\cline { 2 - 2 } & $16.556(2)$ \\
\hline Cell volume $\mathrm{V}, \AA^{3}$ & $253.87(6)$ \\
\hline Formula units per cell Z & 4 \\
\hline Density $\mathrm{D}_{\mathrm{X}}, \mathrm{g} \mathrm{cm}^{-3}$ & $3.5370(8)$ \\
\hline Reliability factor $\mathrm{R}_{\mathrm{B}}$ & 0.0279 \\
\hline FWHM parameters $\mathrm{U}, \mathrm{V}, \mathrm{W}$ & $0.021(4), 0.001(1), 0.0469(6)$ \\
\hline Mixing parameter $\eta$ & $0.864(6)$ \\
\hline Asymmetry parameter $\mathrm{C}_{\mathrm{M}}$ & $-0.051(1)$ \\
\hline Reliability factors $\mathrm{R}_{\mathrm{p}}, \mathrm{R}_{\mathrm{wp}}$ & $0.0563,0.2476$ \\
\hline
\end{tabular}

Table 6

Atomic coordinates and isotropic displacement parameters for $\mathbf{T i}_{1.15} \mathrm{Cu}_{0.09} \mathrm{Al}_{2.76}$

\begin{tabular}{c|c|c|c|c|c}
\hline Site & $\begin{array}{c}\text { Wyckoff } \\
\text { position }\end{array}$ & $\mathrm{x}$ & $\mathrm{y}$ & $\mathrm{z}$ & $\mathrm{B}_{\text {iso }}, \AA^{2}$ \\
\hline $\mathrm{Ti}$ & $4 \mathrm{e}$ & 0 & 0 & $0.1293(3)$ & $0.76(12)$ \\
\hline $\mathrm{Al}$ & $4 \mathrm{~d}$ & 0 & $1 / 2$ & $1 / 4$ & $0.8(2)$ \\
\hline $\mathrm{M} 1^{1}$ & $4 \mathrm{c}$ & 0 & $1 / 2$ & 0 & $0.8(2)$ \\
\hline $\mathrm{M}^{2}$ & $4 \mathrm{e}$ & 0 & 0 & $0.3599(3)$ & $0.6(2)$ \\
\hline
\end{tabular}

Note: ${ }^{1}-\mathrm{M} 1=0.85(2) \mathrm{Al}+0.15(2) \mathrm{Ti} ;{ }^{2}-\mathrm{M} 2=0.91(1) \mathrm{Al}+0.09(1) \mathrm{Cu}$. Microhardness

The microhardness of a substance is an important parameter to define the strength of the material. This property is basically related to the crystal structure of the material or in other words, the way in which the atoms are packed and the electronic factors operating to make the structure. Microhardness values of the four ternary phases of $\mathrm{Ti}-\mathrm{Cu}-\mathrm{Al}$ system investigated here are given in Table 7. 
Table 7

Microhardness of the ternary phases in the Ti-Cu-Al system, compactness (occupied fraction $f)$ density $\left(D_{x}\right)$ and selected interatomic distances (8) from X-ray diffraction

\begin{tabular}{c|c|c|c|c}
\hline Sample & \multicolumn{2}{|c|}{$\mathrm{Ti}_{25} \mathrm{Cu}_{6.25} \mathrm{Al}_{68.75}$} & $\mathrm{Ti}_{25} \mathrm{Cu}_{50} \mathrm{Al}_{25}$ & $\mathrm{Ti}_{33.3} \mathrm{Cu}_{23} \mathrm{Al}_{43.7}$ \\
\hline Phase & $\begin{array}{c}\mathrm{TiCu}_{0.45(4)} \mathrm{Al}_{2.55(4)} \\
\left(\mathrm{Cu}_{3} \mathrm{Au} \text { type }\right)\end{array}$ & $\begin{array}{c}\mathrm{Ti}_{1.15} \mathrm{Cu}_{0.09} \mathrm{Al}_{2.76} \\
\left(\mathrm{ZrAl}_{3} \text { type }\right)\end{array}$ & $\begin{array}{c}\mathrm{TiCu}_{2.11(4)} \mathrm{Al}_{0.89(4)} \\
\left(\mathrm{MnCu}_{2} \mathrm{Al} \text { type }\right)\end{array}$ & $\begin{array}{c}\mathrm{TiCu}_{0.72(3)} \mathrm{Al}_{1.28(3)} \\
\left(\mathrm{MgZn}_{2} \text { type }\right)\end{array}$ \\
\hline Microhardness, GPa & $0.324(5)$ & $0.517(5)$ & $0.524(2)$ & $0.894(6)$ \\
\hline Compactness f, \% & 79 & 79 & 77 & 84 \\
\hline Density $\mathrm{D}_{\mathrm{X}}, \mathrm{g} \mathrm{cm}$ & -3 & 4.344 & 6.097 & 5.019 \\
\hline \multirow{3}{*}{$\delta, \AA$} & 3.679 & $\mathrm{Ti}-\mathrm{M}$ & $\mathrm{Ti}-\mathrm{M}$ & $\mathrm{Ti}-\mathrm{M}$ \\
& $\mathrm{Ti}-\mathrm{M}$ & 2.752 & 2.605 & 2.455 \\
& 2.785 & $\mathrm{M}-\mathrm{M}$ & $\mathrm{M}-\mathrm{M}$ & $\mathrm{M}-\mathrm{M}$ \\
& $\mathrm{M}-\mathrm{M}$ & 2.758 & 2.598 & 2.430 \\
\hline
\end{tabular}

The microhardness of the samples ranges from 0.324 to $0.894 \mathrm{GPa}$. The larger value $(0.894 \mathrm{GPa})$ is for the phase that has the largest $\mathrm{Ti}$ content (sample $\mathrm{Ti}_{33.3} \mathrm{Cu}_{23} \mathrm{Al}_{43.7}$ ). It should be noted that the microhardness does not depend on the atomic weight, but on the coefficient of compactness (occupied fraction, $\mathrm{f}$ ). The microhardness increases to the tetrahedrally close-packed structure $\mathrm{MgZn}_{2}$ which coefficient of compactness (84\%) is larger than in the cubic close-packed structure $\mathrm{Cu}_{3} \mathrm{Au}$ (79\%).

\section{Conclusions}

The isothermal cross-section of the phase diagram of the system $\mathrm{Ti}-\mathrm{Cu}-\mathrm{Al}$ at $800^{\circ} \mathrm{C}$ was constructed. Three purely ternary compounds with relatively large homogeneity ranges corresponding to substitution of $\mathrm{Cu}$ for $\mathrm{Al}$ form. A fourth ternary compound with $\mathrm{ZrAl}_{3}$-type structure was observed near the composition $\mathrm{TiAl}_{3}$. The largest microhardness was observed for the Laves phase.

\section{Acknowledgements}

This work was carried out under the grants of the Ministry of Education and Science of Ukraine No. 0118U003609 and No. 0117U007192. The authors are grateful to Dr. A. Horyn for assistance during the DTA measurements.

\section{REFERENCES}

1. Aluminum-copper-titanium / Ran Q., Stadelmaier H.H., Petzow G., Effenberg G. Verlagsgesellschaft VCH // Ternary Alloys. - 1992. - Vol.5. - P.51-66.

2. Villars P., Cenzual K. Pearson's crystal data, crystal structure database for inorganic compounds, release 2018/19. Materials Park: ASM International, 2018.

3. Villars P., Cenzual K., Gladyshevskii R. Handbook of Inorganic Substances 2017. - Berlin: Walter de Gruyter, 2017. $1955 \mathrm{p}$.

4. Einige Strukturdaten metallischer Phasen (9) / K.
Schubert, H.G. Meissner, A. Raman, W. Rossteutscher // Naturwissenschaften. - 1964. - Vol.51. - P.287.

5. Markiv V.Ya., Burnashchova V.V., Ryabov V.R. Issledovanie sistem $\mathrm{Ti}-\mathrm{Fe}-\mathrm{Al}, \mathrm{Ti}-\mathrm{Ni}-\mathrm{Al} \mathrm{i} \mathrm{Ti}-\mathrm{Cu}-\mathrm{Al} / /$ Metallofizika. - 1973. - Vol.46. - P.103-110.

6. Virdis $P$., Zwicker $U$. Phase equilibria in the coppertitanium-aluminium system // Z. Metallkd. - 1971. - Vol.62. P.46-51.

7. A new antibacterial titanium-copper sintered alloy: Preparation and antibacterial property / Zhang E., Li F., Wang H., Liu J., Wang C., Li M., Yang K. // Mater. Sci. Eng. - 2013. - Vol.33. - P.4280-4287.

8. Bleicher L., Sasaki J.M., Paiva Santos C.O. Development of a graphical interface for the Rietveld refinement program DBWS // J. Appl. Crystallogr. - 2000. - Vol.33. - P.1189.

9. Muon diffusion in intermetallic compounds of the $\mathrm{MoSi}_{2}-$ type structure / Mendes P.J., Ferreira L.P., Gil J.M., De Campos Ayres N. // J. Phys. Condens. Matter. - 2001. - Vol.13. P.5285-5293.

10. Braun J., Ellner M. Phase equilibria investigations on the aluminum-rich part of the binary system $\mathrm{Ti}-\mathrm{Al} / /$ Metall. Mater. Trans. A. - 2001. - Vol.32. - P.1037-1047.

11. High-temperature synchrotron X-ray diffraction study of phases in a gamma TiAl alloy / Novoselova T.V., Malinov S.A., Sha W., Zhecheva A. // Mater. Sci. Eng., A. - 2004. - Vol.371. - P.103-112.

12. Villars P., Okamoto H., Cenzual K. ASM alloy phase diagram database. - Materials Park: ASM International, 2017.

13. Pauling file. Inorganic materials database and design system / Villars P., Cenzual K., Daams J.L.C., Hulliger F., Massalski T.B., Okamoto H., Osaki K., Prince A., Iwata S. Binaries Edition. - Bonn: Crystal Impact, 2001.

14. Bulanova M., Tretyachenko L., Golovkova M. Phase equilibria in the Ti-rich corner of the $\mathrm{Ti}-\mathrm{Si}-\mathrm{Al}$ system // Z. Metallkd. - 1997. - Vol.88. - P.256-265.

15. Hahn T. International tables for crystallography. Dordrecht: Kluwer, 2002. - Vol.A. - 911 p.

Received 7.06.2019 


\section{ПОВТОРНЕ ДОСЛІДЖЕННЯ СИСТЕМИ Ті-Cu-Al ПРИ} $800^{\circ} \mathrm{C}$

\section{Н. Климентій, С. Пукас, Л. Оровчик, Р. Гладишевський}

Ізотермічний переріз діаграми стану системи $\mathrm{Ti}-\mathrm{Cu}-\mathrm{A}$ при $800^{\circ}$ С досліджено рентгенівським методом порошку, мікроструктурним аналізом та енергодисперсійною рентгенівською спектроскопією. Для трьох тернарних сполук визначено області гомогенності при $800^{\circ} \mathrm{C}: \mathrm{TiCu}_{x} \mathrm{Al}_{3-x}, x=0,30(3)-0,60(3)$ (структурний тип $\left.\mathrm{Cu}_{3} \mathrm{Au}\right), \mathrm{TiCu}_{2+x} A l_{1-x}, x=0-0,52(2)$ (структурний тип $\mathrm{MnCu} \mathrm{u}_{2} \mathrm{Al}$ ) та $\mathrm{TiCu} \mathrm{u}_{x} \mathrm{Al} \mathrm{2}_{-x}, x=0,54(3)-1,14(3)$ (структурний тип $\mathrm{MgZn}$ ). Кристалічну структуру додаткової сполуки поблизу складу $\mathrm{TiAl}_{3}$ уточнено з даних рентгенівської порошкової дифракції $\left(\mathrm{Ti}_{1,15(2)} \mathrm{Cu}_{0,09(1)} \mathrm{Al} \mathrm{l}_{2,76(3)}\right.$, структурний тип $\mathrm{ZrAl} \mathrm{l}_{3}$; символ Пірсона tI16, просторова група I4/mmm, $a=3,9159(3)$, $c=16,556(2) \AA)$. Ця тернарна сполука, виявлена при $800^{\circ} \mathrm{C}$, ймовірно є продовженням твердого розчину на основі бінарної високотемпературної фази, наданої як $\mathrm{Ti}_{5} A l_{1 l}$, із аналогічним структурним типом. Поміряно мікротвердість тернарних спо$л у к$.

Ключові слова: алюміній, купрум, титан, рентгенівська порошкова дифракція, діаграма стану, тернарна сполука, мікроструктура, твердість.

\section{REINVESTIGATION OF THE SYSTEM Ti-Cu-Al AT $800^{\circ} \mathrm{C}$} N. Klymentiy ${ }^{a,}$, S. Pukas ${ }^{a}$, L. Orovčik ${ }^{b}$, R. Gladyshevskii ${ }^{a}$

a Ivan Franko National University of Lviv, Lviv, Ukraine

b Institute of Materials and Machine Mechanics, Bratislava, Slovakia

* e-mail: nastasia.klymentiy@Inu.edu.ua

The isothermal section of the phase diagram of the ternary system $\mathrm{Ti}-\mathrm{Cu}-\mathrm{Al}$ at the temperature of $800^{\circ} \mathrm{C}$ was investigated using $X$-ray powder diffraction, microstructural analysis and energydispersive $X$-ray spectroscopy. The homogeneity ranges at $800^{\circ} \mathrm{C}$ of three purely ternary compounds were determined: $\mathrm{TiCu}_{x} A l_{3-x}$ $x=0.30(3)-0.60(3)$ (structure type $\mathrm{Cu}_{3} \mathrm{Au}$ ), $\mathrm{TiCu}_{2+{ }_{x}} A l_{1-x}, x=0-$ $0.52(2)$ (structure type $\mathrm{MnCu} \mathrm{Al}_{2}$ ) and $\mathrm{TiCu} \mathrm{Al}_{2-x}, x=0.54(3)-1.14(3)$ (structure type $\mathrm{MgZn} \mathrm{n}_{2}$ ). The crystal structure of an additional compound near the composition $\mathrm{TiAl}_{3}$ was refined on $X$-ray powder diffraction data $\left(\mathrm{Ti}_{1.15(2)} \mathrm{Cu}_{0.09(1)} \mathrm{Al}_{2.76(3)}\right.$, structure type $\mathrm{ZrAl}_{3}$; Pearson symbol tI16, space group I4/mmm, $a=3.9159(3), c=16.556(2) A)$. This ternary compound, observed at $800^{\circ} \mathrm{C}$, is probably an extension of the solid solution of the binary high-temperature phase reported as $\mathrm{Ti}_{5} \mathrm{Al}_{11}$ with the same structure type. The microhardness of the ternary compounds was measured.

Keywords: aluminum; copper; titanium; X-ray powder diffraction; phase diagram; ternary compound; microstructure; hardness.

\section{REFERENCES}

1. Ran Q., Stadelmaier H.H. Aluminum-copper-titanium. In: Ternary Alloys. Petzow G., Effenberg G. (Eds.). VCH Verlagsgesellschaft, Weinheim, Germany, 1992, vol. 5, pp. 5166.

2. Villars P., Cenzual K., Pearson's crystal data, crystal structure database for inorganic compounds. Release 2018/19. ASM International, Materials Park, 2018.

3. Villars P., Cenzual K., Gladyshevskii R., Handbook of Inorganic Substances 2017. Walter de Gruyter, Berlin, 2017. 1955 p.

4. Schubert K., Meissner H.G., Raman A., Rossteutscher W. Einige Strukturdaten metallischer Phasen (9) Naturwissenschaften, 1964, vol. 51, pp. 287-287. (in German).

5. Markiv V.Ya., Burnashchova V.V., Ryabov V.R. Isledovanie sistem $\mathrm{Ti}-\mathrm{Fe}-\mathrm{Al}, \mathrm{Ti}-\mathrm{Ni}-\mathrm{Al}$ i $\mathrm{Ti}-\mathrm{Cu}-\mathrm{Al}$ [Investigation of the systems $\mathrm{Ti}-\mathrm{Fe}-\mathrm{Al}, \mathrm{Ti}-\mathrm{Ni}-\mathrm{Al}$ and $\mathrm{Ti}-\mathrm{Cu}-\mathrm{Al}$ ]. Metallofizika, 1973, vol. 46, pp. 103-110. (in Russian).

6. Virdis P., Zwicker U. Phase equilibria in the coppertitanium-aluminium system. Zeitschrift fur Metallkunde, 1971, vol. 62 , pp. 46-51.

7. Zhang E., Li F., Wang H., Liu J., Wang C., Li M. Yang K. A new antibacterial titanium-copper sintered alloy: preparation and antibacterial property. Materials Science and Engineering C, 2013, vol. 33, pp. 4280-4287.

8. Bleicher L., Sasaki J.M., Paiva Santos C.O. Development of a graphical interface for the Rietveld refinement program DBWS. Journal of Applied Crystallography, 2000, vol. 33, pp. 1189 1189.

9. Mendes P.J., Ferreira L.P., Gil J.M., De Campos N.A. Muon diffusion in intermetallic compounds of the $\mathrm{MoSi}_{2}$-type structure. Journal of Physics: Condensed Matter, 2001, vol. 13 , article no. 5285 .

10. Braun J., Ellner M. Phase equilibria investigations on the aluminum-rich part of the binary system Ti-Al. Metallurgical and Materials Transactions A: Physical Metallurgy and Materials Science, 2001, vol. 32, pp. 1037-1047.

11. Novoselova T., Malinov S., Sha W., Zhecheva A. Hightemperature synchrotron X-ray diffraction study of phases in a gamma TiAl alloy. Materials Science and Engineering A, 2004, vol. 371, pp. 103-112.

12. Villars P., Okamoto H., Cenzual K., ASM Alloy Phase Diagram Database. ASM International, Materials Park, 2017.

13. Villars P., Cenzual K., Daams J.L.C., Hulliger F., Massalski T.B., Okamoto H., Osaki K., Prince A., Iwata S., Pauling File. Inorganic materials database and design system. Binaries edition. Crystal Impact, Bonn, 2001.

14. Bulanova M., Tretyachenko L., Golovkova M. Phase equilibria in the $\mathrm{Ti}$-rich corner of the $\mathrm{Ti}-\mathrm{Si}-\mathrm{Al}$ system. Zeitschrift fur Metallkunde, 1997, vol. 88, pp. 256-265.

15. Hahn T., International tables for crystallography. Kluwer, Dordrecht, 2002, vol. A. 911 p. 